ESAIM: M2AN 48 (2014) 135-163

DOI: $10.1051 / \mathrm{m} 2 \mathrm{an} / 2013099$
ESAIM: Mathematical Modelling and Numerical Analysis

www.esaim-m2an.org

\title{
ERROR ESTIMATES FOR GALERKIN REDUCED-ORDER MODELS OF THE SEMI-DISCRETE WAVE EQUATION
}

\author{
D. Amsallem ${ }^{1}$ and U. Hetmaniuk ${ }^{2}$
}

\begin{abstract}
Galerkin reduced-order models for the semi-discrete wave equation, that preserve the second-order structure, are studied. Error bounds for the full state variables are derived in the continuous setting (when the whole trajectory is known) and in the discrete setting when the Newmark average-acceleration scheme is used on the second-order semi-discrete equation. When the approximating subspace is constructed using the proper orthogonal decomposition, the error estimates are proportional to the sums of the neglected singular values. Numerical experiments illustrate the theoretical results.
\end{abstract}

Mathematics Subject Classification. 65L20, 65M12, 65M15.

Received March 15, 2012.

Published online December 18, 2013.

\section{INTRODUCTION}

Reduced-order models have received lots of attention for simulating dynamical systems at a reduced cost. Numerical simulations have highlighted the efficiency of model-order reduction for many applications. Examples include linear time-invariant dynamical systems with fixed parameter values [6], systems governed by parameterized partial differential equations (PDE) [18,34,36,37], biology [4], aeroelasticity [1,3,27,35], and structural dynamics $[2,12,13,20]$. Although reduction techniques have been used for many years, theoretical results on the state approximation error remain scarce.

For first-order dynamical systems, Rathinam and Petzold [31] derive a priori error bounds of solutions from a proper orthogonal decomposition (POD) reduced-order model and analyze the effects of small perturbation on the subspace used for constructing the POD basis. In a follow-up work, Homescu, Petzold, and Serban [16] compute estimates and bounds for these errors by a combination of small sample statistical condition estimation and error estimation using the adjoint method. In [21,22], Kunisch and Volkwein derive error estimates for a Galerkin POD-based method for a class of nonlinear parabolic partial differential equations (PDE). Nonlinear problems with a Lipschitz continuous nonlinearity are considered in [21] and extended to the Navier-Stokes equations in [22]. POD-based Galerkin schemes for parabolic partial differential equations are analyzed before any spatial discretization is applied. Discrete systems, obtained after time integration with the backward Euler, the forward Euler, and the Crank-Nicholson methods, are also analyzed. Their analysis provides a rigorous

Keywords and phrases. Model order reduction, proper orthogonal decomposition, wave equation.

1 Department of Aeronautics and Astronautics, Stanford University, Stanford, CA 94305, USA. amsallem@stanford.edu

2 Department of Applied Maths, University of Washington, Box 353925, Seattle, WA 98195-3925, USA. hetmaniu@uw.edu 
argument to the heuristic rule that the error in trajectory approximation should be proportional to the sum of neglected singular values in the truncated singular value decomposition (SVD) used to obtain the reduced basis. Following the lecture notes [38], Chaturantabut and Sorensen [11] study state approximation error of PODreduced systems for sets of coupled ordinary differential equations with Lipschitz continuous nonlinearities. Their theoretical result separates the effect of the model reduction from the effect of time discretization and is illustrated for the case of backward Euler time integration.

Even though the semi-discrete wave equation,

$$
\frac{\mathrm{d}^{2} \mathbf{y}}{\mathrm{d} t^{2}}(t)+\mathbf{C y}(t)=\mathbf{b}(t)
$$

(where $\mathbf{C}$ is a positive matrix), can be formulated as a first-order dynamical system,

$$
\frac{\mathrm{d}}{\mathrm{d} t}\left[\begin{array}{c}
\frac{\mathrm{d} \mathbf{y}}{\mathrm{d} t} \\
\mathbf{y}
\end{array}\right]+\left[\begin{array}{cc}
\mathbf{0} & \mathbf{C} \\
-\mathbf{I} & \mathbf{0}
\end{array}\right]\left[\begin{array}{l}
\frac{\mathrm{d} \mathbf{y}}{\mathrm{d} t} \\
\mathbf{y}
\end{array}\right]=\left[\begin{array}{l}
\mathbf{f} \\
\mathbf{0}
\end{array}\right]
$$

model-order reduction for the first-order system (1.2) might destroy the original structure of (1.1): a reducedorder system for (1.2) may not correspond to a second-order system of the form (1.1) and the final reduced state variables may not retain a physical interpretation (for example, by mixing values of the state and of its time-derivative). Beattie and Gugercin $[7,8]$ argue that, to preserve the structure, the model-order reduction should be applied directly to the second-order system. Recently, Chapelle, Gariah, and Sainte-Marie [10] derived error estimates for a Galerkin-POD reduced system in a function space setting for the wave equation. Their error analysis relies on a specific projection operator to prove new estimates for the continuous solution. The authors do not consider time discretization issues. Herkt et al. [14] present an extension for the error analysis of Kunisch and Volkwein [21,22] to Galerkin-based reduced systems arising from the second-order semi-discretized wave equation. They study two sets of snapshots. When the snapshots are composed only of state variables (or displacements), their result yields an upper estimate that grows with decreasing time steps. To control the right hand side of their error estimate, Herkt et al. [14] include, in the snapshots set, all the velocities and accelerations. The objective of this paper is to derive error estimates for Galerkin-based reduced systems applied to the second-order semi-discretized wave equation using a first-order formulation that still preserves the original structure. The resulting estimates remain bounded with decreasing time steps for Galerkin-based reduced systems that preserve the structure of the wave equation.

The paper is organized as follows. Section 2 reviews useful general error estimates for a first-order system,

$$
\frac{\mathrm{d} \mathbf{y}}{\mathrm{d} t}(t)=\mathbf{A y}(t)+\mathbf{b}(t) .
$$

Then new error estimates are presented for the discrete system obtained after time integration with the Crank-Nicholson scheme, also investigated in $[23,32]$. These results are extended to generalized systems of the form,

$$
\mathcal{M} \frac{\mathrm{d} \mathbf{x}}{\mathrm{d} t}(t)=\mathcal{A} \mathbf{x}(t)+\mathbf{f}(t)
$$

by selecting the appropriate norms to measure the errors. Section 3 presents new estimates for the semi-discrete wave equation,

$$
\mathbf{M} \frac{\mathrm{d}^{2} \mathbf{u}}{\mathrm{d} t^{2}}(t)+\mathbf{D} \frac{\mathrm{d} \mathbf{u}}{\mathrm{d} t}(t)+\mathbf{K u}(t)=\mathbf{b}(t),
$$

while preserving the structure of the second-order system. The discrete system obtained after time integration with the average constant acceleration Newmark scheme $\left(\gamma=\frac{1}{2}, \beta=\frac{1}{4}\right)$ is analyzed. Finally Section 4 describes numerical experiments highlighting the theoretical results. For the sake of clarity, most of the proofs are gathered in the Appendix. 


\section{ERror ESTIMATES FOR A SYSTEM FOR FIRST-ORDER ORDINARY DIFFERENTIAL EQUATIONS}

This section recalls several standard theoretical results that will be useful for our analysis. These results provide bounds on the state errors when approximating the solution of a full-order model with the solution of a reduced-order model.

\subsection{Error estimates for a simple first-order dynamical system}

Consider the system

$$
\frac{\mathrm{d} \mathbf{y}}{\mathrm{d} t}(t)=\mathbf{A y}(t)+\mathbf{b}(t), \quad \mathbf{y}(0)=\mathbf{y}_{0}, \quad \text { for } t \in[0, T]
$$

where the matrix $\mathbf{A}$ is constant. Such system appears, for example, after spatial discretization of a parabolic partial differential equation (as studied in [21]). Results for the system (2.1) are a compilation of bounds from the works $[11,21,31]$.

To build a reduced-order model by Galerkin projection on a subspace, a matrix $\mathbf{V}$ is introduced with columns spanning the subspace. The approximation of state variables $\mathbf{y}$ is defined as

$$
\mathbf{y}_{\mathrm{ROM}}(t)=\tilde{\mathbf{y}}(t)+\mathbf{V} \mathbf{y}_{r}(t),
$$

where $\tilde{\mathbf{y}}(t)$ is a known function ${ }^{3}$, and

$$
\mathbf{V}^{T}\left(\frac{\mathrm{d} \mathbf{y}_{\mathrm{ROM}}}{\mathrm{d} t}(t)-\mathbf{A} \mathbf{V y}_{\mathrm{ROM}}(t)-\mathbf{b}(t)\right)=\mathbf{0} .
$$

The reduced variables $\mathbf{y}_{r}$ are defined by the evolution equation

$$
\left\{\begin{aligned}
\frac{\mathrm{d} \mathbf{y}_{r}}{\mathrm{~d} t}(t) & =\left(\mathbf{V}^{T} \mathbf{V}\right)^{-1} \mathbf{V}^{T} \mathbf{A} \mathbf{V} \mathbf{y}_{r}(t)+\left(\mathbf{V}^{T} \mathbf{V}\right)^{-1} \mathbf{V}^{T}\left(\mathbf{b}+\mathbf{A} \tilde{\mathbf{y}}(t)-\frac{\mathrm{d} \tilde{\mathbf{y}}}{\mathrm{d} t}(t)\right), \\
\mathbf{y}_{r}(0) & =\left(\mathbf{V}^{T} \mathbf{V}\right)^{-1} \mathbf{V}^{T}\left(\mathbf{y}_{0}-\tilde{\mathbf{y}}(0)\right),
\end{aligned}\right.
$$

over the time interval $[0, T]$.

The error is defined as

$$
\begin{aligned}
\mathbf{e}_{\mathrm{ROM}}(t)=\mathbf{y}(t)-\tilde{\mathbf{y}}(t)-\mathbf{V y}_{r}(t)=\mathbf{y}(t)-\tilde{\mathbf{y}}(t)-\mathbf{V}\left(\mathbf{V}^{T} \mathbf{V}\right)^{-1} & \mathbf{V}^{T}(\mathbf{y}(t)-\tilde{\mathbf{y}}(t)) \\
& +\mathbf{V}\left[\left(\mathbf{V}^{T} \mathbf{V}\right)^{-1} \mathbf{V}^{T}(\mathbf{y}(t)-\tilde{\mathbf{y}}(t))-\mathbf{y}_{r}(t)\right]
\end{aligned}
$$

and decomposed into two parts, $\mathbf{e}_{\mathrm{ROM}}=\mathbf{e}_{P}+\mathbf{e}_{V}$, where $\mathbf{e}_{P}$ is the error arising from the projection into the subspace spanned by $\mathbf{V}$

$$
\mathbf{e}_{P}(t)=\mathbf{y}(t)-\tilde{\mathbf{y}}(t)-\mathbf{V}\left(\mathbf{V}^{T} \mathbf{V}\right)^{-1} \mathbf{V}^{T}(\mathbf{y}(t)-\tilde{\mathbf{y}}(t))
$$

and $\mathbf{e}_{V}$ is the error inside the subspace

$$
\mathbf{e}_{V}(t)=\mathbf{V}\left[\left(\mathbf{V}^{T} \mathbf{V}\right)^{-1} \mathbf{V}^{T}(\mathbf{y}(t)-\tilde{\mathbf{y}}(t))-\mathbf{y}_{r}(t)\right]
$$

The error $\mathbf{e}_{V}$ satisfies the equation

$$
\frac{\mathrm{d} \mathbf{e}_{V}}{\mathrm{~d} t}=\mathbf{V}\left[\left(\mathbf{V}^{T} \mathbf{V}\right)^{-1} \mathbf{V}^{T} \frac{\mathrm{d} \mathbf{y}}{\mathrm{d} t}-\left(\mathbf{V}^{T} \mathbf{V}\right)^{-1} \mathbf{V}^{T} \frac{\mathrm{d} \tilde{\mathbf{y}}}{\mathrm{d} t}-\frac{\mathrm{d} \mathbf{y}_{r}}{\mathrm{~d} t}\right]=\mathbf{V}\left(\mathbf{V}^{T} \mathbf{V}\right)^{-1} \mathbf{V}^{T} \mathbf{A}\left(\mathbf{e}_{P}+\mathbf{e}_{V}\right)
$$

\footnotetext{
${ }^{3}$ Usually, $\tilde{\mathbf{y}}$ is chosen constant and equal to $\mathbf{0}$ or $\mathbf{y}_{0}$. A time-dependent choice does not impact the analysis and such a choice will be convenient for the second-order wave equation.
} 
with the initial condition $\mathbf{e}_{V}(0)=\mathbf{0}$. The explicit formula for $\mathbf{e}_{V}$ is

$$
\begin{aligned}
\mathbf{e}_{V}(t)=\mathbf{V} \int_{0}^{t} \mathrm{e}^{\left(\mathbf{V}^{T} \mathbf{V}\right)^{-1} \mathbf{V}^{T} \mathbf{A} \mathbf{V}(t-\tau)} & \left(\mathbf{V}^{T} \mathbf{V}\right)^{-1} \mathbf{V}^{T} \mathbf{A} \mathbf{e}_{P}(\tau) \mathrm{d} \tau \\
& =\mathbf{V} \int_{0}^{t} \mathrm{e}^{\left(\mathbf{V}^{T} \mathbf{V}\right)^{-1} \mathbf{V}^{T} \mathbf{A} \mathbf{V}(t-\tau)}\left(\mathbf{V}^{T} \mathbf{V}\right)^{-1} \mathbf{V}^{T} \mathbf{A} \mathbf{Z}\left(\mathbf{Z}^{T} \mathbf{Z}\right)^{-1} \mathbf{Z}^{T} \mathbf{e}_{P}(\tau) \mathrm{d} \tau
\end{aligned}
$$

where $\mathbf{Z}$ denotes a matrix whose columns span the orthogonal complement of the span of $\mathbf{V}^{4}$.

Theorem 2.1. The error between the solution $\mathbf{y}$ and the approximation $\mathbf{y}_{\mathrm{ROM}}$ satisfies

$$
\begin{aligned}
\int_{0}^{T}\left\|\mathbf{e}_{\mathrm{ROM}}(t)\right\|_{2}^{2} \mathrm{~d} t \leq\left(\| F\left(T,\left(\mathbf{V}^{T} \mathbf{V}\right)^{-1} \mathbf{V}^{T} \mathbf{A} \mathbf{V}\right)\right. & \left.\left\|_{\mathbf{V}^{T} \mathbf{V}}^{2}\right\| \mathbf{V}^{T} \mathbf{A} \mathbf{Z}\left(\mathbf{Z}^{T} \mathbf{Z}\right)^{-1} \mathbf{Z}^{T} \|_{\left(\mathbf{V}^{T} \mathbf{V}\right)^{-1}}^{2}+1\right) \\
& \int_{0}^{T}\left\|\mathbf{y}(t)-\tilde{\mathbf{y}}(t)-\mathbf{V}\left(\mathbf{V}^{T} \mathbf{V}\right)^{-1} \mathbf{V}^{T}(\mathbf{y}(t)-\tilde{\mathbf{y}}(t))\right\|_{2}^{2} \mathrm{~d} t
\end{aligned}
$$

where $\|\cdot\|_{2}$ is the usual Euclidian norm. When $\mathbf{H}$ is a symmetric positive definite matrix, the matrix norm $\|\mathbf{B}\|_{\mathbf{H}}$ is $\sqrt{\lambda_{\max }\left(\mathbf{B}^{T} \mathbf{H B}\right)}$. The constant $\|F(T, \mathbf{B})\|_{\mathbf{H}}$ is defined by

$$
\|F(T, \mathbf{B})\|_{\mathbf{H}}=\sup _{\mathbf{u} \neq \mathbf{0}} \sqrt{\frac{\int_{0}^{T}\left\|\int_{0}^{t} \mathrm{e}^{\mathbf{B}(t-\tau)} \mathbf{u}(\tau) \mathrm{d} \tau\right\|_{\mathbf{H}}^{2} \mathrm{~d} t}{\int_{0}^{T}\|\mathbf{u}(t)\|_{\mathbf{H}}^{2} \mathrm{~d} t}} .
$$

This result appeared in [11,31]. The amplification factor in front of the projection error depends on the original problem through the quantities $\mathbf{A}$ and $T$ as well as the reduced-order model through $\mathbf{V}$. The constant $\left\|F\left(T,\left(\mathbf{V}^{T} \mathbf{V}\right)^{-1} \mathbf{V}^{T} \mathbf{A V}\right)\right\|_{\mathbf{V}^{T} \mathbf{V}}$ is discussed in [11,31]. It usually grows or decays exponentially with $T$ at a rate that is proportional to the largest eigenvalue for the pencil $\left(\mathbf{V}^{T}\left(\mathbf{A}^{T}+\mathbf{A}\right) \mathbf{V}, \mathbf{V}^{T} \mathbf{V}\right)$. The bound (2.10) indicates that the error $\mathbf{e}_{\mathrm{ROM}}$ is controlled by the error from the projection into the subspace spanned by $\mathbf{V}$.

If the entire continuous trajectory $\mathbf{y}(t)$ is available on the interval $[0, T]$, a basis generated by the proper orthogonal decomposition $[19,28]$ satisfies the following minimization problem

$$
\min _{\operatorname{rank}\{\mathbf{V}\}=k} \int_{0}^{T}\left\|\mathbf{y}(t)-\tilde{\mathbf{y}}(t)-\mathbf{V}\left(\mathbf{V}^{T} \mathbf{V}\right)^{-1} \mathbf{V}^{T}(\mathbf{y}(t)-\tilde{\mathbf{y}}(t))\right\|_{2}^{2} \mathrm{~d} t
$$

and

$$
\int_{0}^{T}\left\|\mathbf{y}(t)-\tilde{\mathbf{y}}(t)-\mathbf{V}\left(\mathbf{V}^{T} \mathbf{V}\right)^{-1} \mathbf{V}^{T}(\mathbf{y}(t)-\tilde{\mathbf{y}}(t))\right\|_{2}^{2} \mathrm{~d} t=\sum_{i=k+1}^{r} \lambda_{i}^{\infty}
$$

where $r$ is the rank for the matrix

$$
\mathbf{R}=\int_{0}^{T}(\mathbf{y}(t)-\tilde{\mathbf{y}}(t))(\mathbf{y}(t)-\tilde{\mathbf{y}}(t))^{T} \mathrm{~d} t
$$

and $\left(\lambda_{i}^{\infty}\right)_{1 \leq i \leq r}$ is the set of non-zero non-increasing eigenvalues for the pencil $(\mathbf{R}, \mathbf{I})$. The notation for $\mathbf{R}$ and for its eigenvalues $\lambda_{i}^{\infty}$ appeared previously in [11,22]. When combined with (2.13), the bound (2.10) indicates that the error when approximating the state variables over the whole time interval $[0, T]$ is proportional to the sum of the neglected singular values obtained from the proper orthogonal decomposition.

\footnotetext{
${ }^{4} \mathbf{V}$ and $\mathbf{Z}$ are often composed of orthonormal columns. The general case without orthonormal columns will be used for analyzing the second-order wave equation.
} 
Remark 2.2. When the matrix $\mathbf{A}$ is symmetric and $\mathbf{V}$ is spanned by a subset of eigenvectors, the term $\mathbf{V}^{T} \mathbf{A Z}$ is $\mathbf{0}$ and the error estimate (2.10) simplifies to

$$
\int_{0}^{T}\left\|\mathbf{e}_{\mathrm{ROM}}(t)\right\|_{2}^{2} \mathrm{~d} t \leq \int_{0}^{T}\left\|\mathbf{y}(t)-\tilde{\mathbf{y}}(t)-\mathbf{V}\left(\mathbf{V}^{T} \mathbf{V}\right)^{-1} \mathbf{V}^{T}(\mathbf{y}(t)-\tilde{\mathbf{y}}(t))\right\|_{2}^{2} \mathrm{~d} t .
$$

Next consider the discrete system obtained when using the trapezoidal time integration scheme,

$$
\frac{\mathbf{y}_{j}-\mathbf{y}_{j-1}}{\Delta t}=\mathbf{A}\left(\frac{\mathbf{y}_{j}+\mathbf{y}_{j-1}}{2}\right)+\frac{\mathbf{b}_{j}+\mathbf{b}_{j-1}}{2}, \text { for } 1 \leq j \leq n_{t},
$$

where $\Delta t=T / n_{t}, \mathbf{b}_{j}=\mathbf{b}(j \Delta t)$ and $\mathbf{y}_{j}$ is the approximation of $\mathbf{y}(j \Delta t)$. Approximating $\mathbf{y}_{j}$ as follows

$$
\mathbf{y}_{j} \approx \tilde{\mathbf{y}}_{j}+\mathbf{V} \hat{\mathbf{y}}_{j}
$$

(where the sequence $\left\{\tilde{\mathbf{y}}_{j}\right\}$ is known a priori), the reduced variables for the incremental update satisfies

$$
\begin{array}{r}
\frac{\hat{\mathbf{y}}_{j}-\hat{\mathbf{y}}_{j-1}}{\Delta t}=\left(\mathbf{V}^{T} \mathbf{V}\right)^{-1} \mathbf{V}^{T} \mathbf{A V}\left(\frac{\hat{\mathbf{y}}_{j}+\hat{\mathbf{y}}_{j-1}}{2}\right)+\left(\mathbf{V}^{T} \mathbf{V}\right)^{-1} \mathbf{V}^{T}\left(\frac{\mathbf{b}_{j}+\mathbf{b}_{j-1}}{2}\right) \\
+\left(\mathbf{V}^{T} \mathbf{V}\right)^{-1} \mathbf{V}^{T} \mathbf{A}\left(\frac{\tilde{\mathbf{y}}_{j}+\tilde{\mathbf{y}}_{j-1}}{2}\right)-\left(\mathbf{V}^{T} \mathbf{V}\right)^{-1} \mathbf{V}^{T}\left(\frac{\tilde{\mathbf{y}}_{j}-\tilde{\mathbf{y}}_{j-1}}{\Delta t}\right)
\end{array}
$$

with

$$
\hat{\mathbf{y}}_{0}=\left(\mathbf{V}^{T} \mathbf{V}\right)^{-1} \mathbf{V}^{T}\left(\mathbf{y}_{0}-\tilde{\mathbf{y}}(0)\right)
$$

Define the error

$$
\mathbf{e}_{j}=\mathbf{y}_{j}-\tilde{\mathbf{y}}_{j}-\mathbf{V} \hat{\mathbf{y}}_{j}=\mathbf{y}_{j}-\tilde{\mathbf{y}}_{j}-\mathbf{V}\left(\mathbf{V}^{T} \mathbf{V}\right)^{-1} \mathbf{V}^{T}\left(\mathbf{y}_{j}-\tilde{\mathbf{y}}_{j}\right)+\mathbf{V}\left[\left(\mathbf{V}^{T} \mathbf{V}\right)^{-1} \mathbf{V}^{T}\left(\mathbf{y}_{j}-\tilde{\mathbf{y}}_{j}\right)-\hat{\mathbf{y}}_{j}\right]
$$

and the decomposition, $\mathbf{e}_{j}=\rho_{j}+\theta_{j}$, where

$$
\rho_{j}=\mathbf{y}_{j}-\tilde{\mathbf{y}}_{j}-\mathbf{V}\left(\mathbf{V}^{T} \mathbf{V}\right)^{-1} \mathbf{V}^{T}\left(\mathbf{y}_{j}-\tilde{\mathbf{y}}_{j}\right) \in \operatorname{span} \mathbf{Z}
$$

and

$$
\theta_{j}=\mathbf{V}\left[\left(\mathbf{V}^{T} \mathbf{V}\right)^{-1} \mathbf{V}^{T}\left(\mathbf{y}_{j}-\tilde{\mathbf{y}}_{j}\right)-\hat{\mathbf{y}}_{j}\right]
$$

Analogously to the previous error $\mathbf{e}_{V}$, the part $\theta_{j}$ satisfies

$$
\frac{\theta_{j}-\theta_{j-1}}{\Delta t}=\mathbf{V}\left(\mathbf{V}^{T} \mathbf{V}\right)^{-1} \mathbf{V}^{T} \mathbf{A}\left(\frac{\rho_{j}+\rho_{j-1}}{2}+\frac{\theta_{j}+\theta_{j-1}}{2}\right)
$$

with $\theta_{0}=\mathbf{0}$.

Theorem 2.3. The norms for the error between the approximation $\mathbf{y}_{j}$ and the discrete solution from the reduced order model (2.17) satisfy

$$
\Delta t \sum_{j=0}^{n_{t}}\left\|\mathbf{y}_{j}-\tilde{\mathbf{y}}_{j}-\mathbf{V} \hat{\mathbf{y}}_{j}\right\|_{2}^{2} \leq\left(\Delta t \sum_{j=0}^{n_{t}}\left\|\mathbf{y}_{j}-\tilde{\mathbf{y}}_{j}-\mathbf{V}\left(\mathbf{V}^{T} \mathbf{V}\right)^{-1} \mathbf{V}^{T}\left(\mathbf{y}_{j}-\tilde{\mathbf{y}}_{j}\right)\right\|_{2}^{2}\right)\left[1+2 \alpha^{2} T\left(\Delta t \sum_{j=0}^{n_{t}} \mathrm{e}^{2 \gamma j \Delta t}\right)\right]
$$

where the coefficient $\alpha$ is

$$
\alpha=\frac{1}{2}\left\|\left(\mathbf{I}-\frac{\Delta t}{2} \mathbf{V}\left(\mathbf{V}^{T} \mathbf{V}\right)^{-1} \mathbf{V}^{T} \mathbf{A}\right)^{-1} \mathbf{V}\left(\mathbf{V}^{T} \mathbf{V}\right)^{-1} \mathbf{V}^{T} \mathbf{A} \mathbf{Z}\left(\mathbf{Z}^{T} \mathbf{Z}\right)^{-1} \mathbf{Z}^{T}\right\|_{2}
$$


and the coefficient $\gamma$ is

$$
\gamma=\left\|\left(\mathbf{I}-\frac{\Delta t}{2} \mathbf{V}\left(\mathbf{V}^{T} \mathbf{V}\right)^{-1} \mathbf{V}^{T} \mathbf{A}\right)^{-1} \mathbf{V}\left(\mathbf{V}^{T} \mathbf{V}\right)^{-1} \mathbf{V}^{T} \mathbf{A}\right\|_{2} .
$$

The proof for this theorem is described in the appendix. This result does not make any assumption on the matrix A, while the analysis of Kunisch and Volkwein ([21], Thm. 8) requires that the continuous counterpart to $\mathbf{A}$ be symmetric negative definite. When the time-step $\Delta t$ goes to 0 , the right hand side of (2.19) remains bounded as

$$
\alpha \rightarrow \frac{1}{2}\left\|\mathbf{V}\left(\mathbf{V}^{T} \mathbf{V}\right)^{-1} \mathbf{V}^{T} \mathbf{A} \mathbf{Z}\left(\mathbf{Z}^{T} \mathbf{Z}\right)^{-1} \mathbf{Z}^{T}\right\|_{2}
$$

and

$$
\Delta t \sum_{j=0}^{n_{t}} \mathrm{e}^{2 \gamma j \Delta t} \rightarrow \int_{0}^{T} \mathrm{e}^{2 \gamma_{*} t} \mathrm{~d} t \quad \text { with } \quad \gamma_{*}=\left\|\mathbf{V}\left(\mathbf{V}^{T} \mathbf{V}\right)^{-1} \mathbf{V}^{T} \mathbf{A}\right\|_{2} .
$$

The proper orthogonal decomposition using the method of snapshots [33] on the snapshots matrix

$$
\hat{\mathbf{S}}=\left[\mathbf{y}_{0}-\tilde{\mathbf{y}}_{0}, \ldots, \mathbf{y}_{n_{t}}-\tilde{\mathbf{y}}_{n_{t}}\right]
$$

can generate a matrix $\mathbf{V}$ whose columns are the eigenvectors of $\hat{\mathbf{S}} \hat{\mathbf{S}}^{T}$ for the $k$ dominant eigenvalues $\left(\lambda_{i}\right)_{1 \leq i \leq r}$. Then the projection error is given by

$$
\sum_{j=0}^{n_{t}}\left\|\mathbf{y}_{j}-\tilde{\mathbf{y}}_{j}-\mathbf{V}\left(\mathbf{V}^{T} \mathbf{V}\right)^{-1} \mathbf{V}^{T}\left(\mathbf{y}_{j}-\tilde{\mathbf{y}}_{j}\right)\right\|_{2}^{2}=\sum_{j=k+1}^{r} \lambda_{i},
$$

where $r$ is the rank of $\hat{\mathbf{S}} \hat{\mathbf{S}}^{T}$.

Remark 2.4. Convergence of the trapezoidal scheme [30] indicates that

$$
\max _{0 \leq j \leq n_{t}}\left\|\mathbf{y}\left(t_{j}\right)-\mathbf{y}_{j}\right\|=\mathcal{O}\left(\Delta t^{2}\right) .
$$

Theorem 2.3 relates the error between the discrete solution from the reduced order model $\left(\tilde{\mathbf{y}}_{j}+\mathbf{V} \hat{\mathbf{y}}_{j}\right)_{0 \leq j \leq n_{t}}$ and the approximation to the exact solution $\left(\mathbf{y}_{j}\right)_{0 \leq j \leq n_{t}}$. The error between the exact solution $\left(\mathbf{y}\left(t_{j}\right)\right)_{0 \leq j \leq n_{t}}$ and the discrete solution from the reduced order model satisfies

$$
\Delta t \sum_{j=0}^{n_{t}}\left\|\mathbf{y}\left(t_{j}\right)-\tilde{\mathbf{y}}_{j}-\mathbf{V} \hat{\mathbf{y}}_{j}\right\|_{2}^{2} \leq 2 \Delta t \sum_{j=0}^{n_{t}}\left\|\mathbf{y}\left(t_{j}\right)-\mathbf{y}_{j}\right\|^{2}+2 \Delta t \sum_{j=0}^{n_{t}}\left\|\mathbf{y}_{j}-\tilde{\mathbf{y}}_{j}-\mathbf{V} \hat{\mathbf{y}}_{j}\right\|_{2}^{2} .
$$

When the time-step $\Delta t$ goes to 0 , both terms on the right hand side will be controlled.

\subsection{Error estimates for a generalized first-order dynamical system}

Consider a generalized first-order dynamical system of the form

$$
\mathcal{M} \frac{\mathrm{d} \mathbf{x}}{\mathrm{d} t}(t)=\mathcal{A} \mathbf{x}(t)+\mathbf{f}(t)
$$

where $\mathcal{M}$ is a symmetric positive definite matrix. Error estimates when approximating the continuous trajectory $\mathbf{x}(t)$ and after time-discretization are presented in this section. The originality of this analysis arises from the transformation between the generalized first-order system (2.1) and the initial system (2.22). This transformation is important because it may have severe implications during the numerical computations. For example, Amsallem and Farhat [5] show that an inappropriate transformation (multliplying by $\mathcal{M}^{-1}$ versus working with $\mathcal{M}^{\frac{1}{2}}$ ) can 
result in unphysical unstable modes of the resulting reduced-order model. Similarly, an inappropriate choice can result in more computational work for an iterative eigensolver [15]. In both cases, the transformation with $\mathcal{M}^{\frac{1}{2}}$ is recommended. This transformation is used here to analyze the system (2.22).

An approximation of $\mathbf{x}$ is constructed in the subspace spanned by the columns of $\mathcal{V}$ such that

$$
\mathbf{x}_{\mathrm{ROM}}(t)=\tilde{\mathbf{x}}(t)+\mathcal{V} \mathbf{x}_{r}(t)
$$

where the function $\tilde{\mathbf{x}}$ is known a priori and the reduced variable $\mathbf{x}_{r}$ is solution to the system

$$
\left\{\begin{aligned}
\mathcal{V}^{T} \mathcal{M} \mathcal{V} \frac{\mathrm{d} \mathbf{x}_{r}}{\mathrm{~d} t}(t) & =\mathcal{V}^{T} \mathcal{A} \mathcal{V} \mathbf{x}_{r}(t)+\mathcal{V}^{T}\left(\mathcal{A} \tilde{\mathbf{x}}(t)+\mathbf{f}(t)-\mathcal{M} \frac{\mathrm{d} \tilde{\mathbf{x}}}{\mathrm{d} t}(t)\right), \\
\mathbf{x}_{r}(0) & =\left(\mathcal{V}^{T} \mathcal{M} \mathcal{V}\right)^{-1} \mathcal{V}^{T} \mathcal{M}\left(\mathbf{x}_{0}-\tilde{\mathbf{x}}(0)\right),
\end{aligned}\right.
$$

over the time interval $[0, T]$.

Define the error $\mathcal{E}_{\mathrm{ROM}}=\mathbf{x}-\mathbf{x}_{\mathrm{ROM}}$ with the parts

$$
\mathcal{E}_{P}=\mathbf{x}-\tilde{\mathbf{x}}-\mathcal{V}\left(\mathcal{V}^{T} \mathcal{M V}\right)^{-1} \mathcal{V}^{T} \mathcal{M}(\mathbf{x}-\tilde{\mathbf{x}})
$$

and

When decomposing $\mathbb{R}^{n}$ as

$$
\mathcal{E}_{\mathcal{V}}=\mathcal{V}\left[\left(\mathcal{V}^{T} \mathcal{M V}\right)^{-1} \mathcal{V}^{T} \mathcal{M}(\mathbf{x}-\tilde{\mathbf{x}})-\mathbf{x}_{r}\right]
$$

$$
\mathbb{R}^{n}=\operatorname{span} \mathcal{V} \oplus^{\perp \mathcal{M}} \operatorname{span} \mathcal{Z}
$$

the projection error $\mathcal{E}_{P}$ belongs to span $\mathcal{Z}$. Theorem 2.1 is modified as follows.

Theorem 2.5. The error between the solution $\mathrm{x}$ of (2.22) and the approximation $\mathrm{x}_{\mathrm{ROM}}$ satisfies

$$
\begin{aligned}
\int_{0}^{T}\left\|\mathcal{E}_{\mathrm{ROM}}(t)\right\|_{\mathcal{M}}^{2} \mathrm{~d} t \leq\left(\| F\left(T,\left(\mathcal{V}^{T} \mathcal{M V}\right)^{-1}\right.\right. & \left.\left.\mathcal{V}^{T} \mathcal{A} \mathcal{V}\right)\left\|_{\mathcal{V}^{T} \mathcal{M V}}^{2}\right\| \mathcal{V}^{T} \mathcal{A} \mathcal{Z}\left(\mathcal{Z}^{T} \mathcal{M} \mathcal{Z}\right)^{-1} \mathcal{Z}^{T} \mathcal{M}^{\frac{1}{2}} \|_{\left(\mathcal{V}^{T} \mathcal{M} \mathcal{V}\right)^{-1}}^{2}+1\right) \\
& \times \int_{0}^{T}\left\|\mathbf{x}(t)-\tilde{\mathbf{x}}(t)-\mathcal{V}\left(\mathcal{V}^{T} \mathcal{M} \mathcal{V}\right)^{-1} \mathcal{V}^{T} \mathcal{M}(\mathbf{x}(t)-\tilde{\mathbf{x}}(t))\right\|_{\mathcal{M}}^{2} \mathrm{~d} t
\end{aligned}
$$

where the constant $\left\|F\left(T,\left(\mathcal{V}^{T} \mathcal{M V}\right)^{-1} \mathcal{V}^{T} \mathcal{A} \mathcal{V}\right)\right\|_{\mathcal{V}^{T} \mathcal{M V}}$ is defined by (2.11).

The constant $\left\|F\left(T,\left(\mathcal{V}^{T} \mathcal{M V}\right)^{-1} \mathcal{V}^{T} \mathcal{A} \mathcal{V}\right)\right\|_{\mathcal{V}^{T} \mathcal{M V}}$ also grows or decays exponentially with $T$ at a rate that is proportional to the largest eigenvalue for the pencil $\left(\mathcal{V}^{T}\left(\mathcal{A}^{T}+\mathcal{A}\right) \mathcal{V}, \mathcal{V}^{T} \mathcal{M V}\right)$. The proof for this theorem relies on the change of variables $\mathbf{y}=\mathcal{M}^{\frac{1}{2}} \mathbf{x}$ and is described in the Appendix.

If the entire continuous trajectory $\mathbf{x}(t)$ is available on the interval $[0, T]$, an approximating subspace may be obtained by solving the minimization problem

$$
\operatorname{argmin}_{\operatorname{rank}\{\mathcal{V}\}=k} \int_{0}^{T}\left\|\mathbf{x}(t)-\tilde{\mathbf{x}}(t)-\mathcal{V}\left(\mathcal{V}^{T} \mathcal{M} \mathcal{V}\right)^{-1} \mathcal{V}^{T} \mathcal{M}(\mathbf{x}(t)-\tilde{\mathbf{x}}(t))\right\|_{\mathcal{M}}^{2} \mathrm{~d} t .
$$

Introducing the matrix

$$
\mathbf{R}=\int_{0}^{T}(\mathbf{x}(t)-\tilde{\mathbf{x}}(t))(\mathbf{x}(t)-\tilde{\mathbf{x}}(t))^{T} \mathrm{~d} t,
$$

an optimal matrix $\mathcal{V}$ of rank $k$ will span a stable subspace for the pencil $(\mathcal{M} \mathbf{R} \mathcal{M}, \mathcal{M})$ (see the Appendix). Denoting $\left(\lambda_{i}^{\infty}\right)_{1 \leq i \leq r}$ the non-zero eigenvalues of the pencil $(\mathcal{M} \mathbf{R} \mathcal{M}, \mathcal{M})$ - ordered in a non-increasing fashion - the projection error for the minimization problem becomes

$$
\min _{\operatorname{rank}\{\mathcal{V}\}=k} \int_{0}^{T}\left\|\mathbf{x}(t)-\tilde{\mathbf{x}}(t)-\mathcal{V}\left(\mathcal{V}^{T} \mathcal{M V}\right)^{-1} \mathcal{V}^{T} \mathcal{M}(\mathbf{x}(t)-\tilde{\mathbf{x}}(t))\right\|_{\mathcal{M}}^{2} \mathrm{~d} t=\sum_{i=k+1}^{r} \lambda_{i}^{\infty} .
$$


Remark 2.6. When the matrix $\mathcal{A}$ is symmetric and $\mathcal{V}$ is spanned by a subset of generalized eigenvectors for the pencil $(\mathcal{A}, \mathcal{M})$, the term $\mathcal{V}^{T} \mathcal{A Z}$ becomes $\mathbf{0}$ and the error estimate (2.26) simplifies to

$$
\int_{0}^{T}\left\|\mathcal{E}_{\mathrm{ROM}}(t)\right\|_{\mathcal{M}}^{2} \mathrm{~d} t \leq \int_{0}^{T}\left\|\mathbf{x}(t)-\tilde{\mathbf{x}}(t)-\mathcal{V}\left(\mathcal{V}^{T} \mathcal{M} \mathcal{V}\right)^{-1} \mathcal{V}^{T} \mathcal{M}(\mathbf{x}(t)-\tilde{\mathbf{x}}(t))\right\|_{\mathcal{M}}^{2} \mathrm{~d} t .
$$

Consider the discrete system obtained from trapezoidal time integration

$$
\mathcal{M}\left(\frac{\mathbf{x}_{j}-\mathbf{x}_{j-1}}{\Delta t}\right)=\mathcal{A}\left(\frac{\mathbf{x}_{j}+\mathbf{x}_{j-1}}{2}\right)+\frac{\mathbf{f}_{j}+\mathbf{f}_{j-1}}{2}, \quad \text { for } 1 \leq j \leq n_{t},
$$

where $\Delta t=T / n_{t}, \mathbf{f}_{j}=\mathbf{f}(j \Delta t)$ and $\mathbf{x}_{j}$ is the approximation of $\mathbf{x}(j \Delta t)$.

A reduced-order model is built for the approximation

$$
\mathbf{x}_{j} \approx \tilde{\mathbf{x}}_{j}+\mathcal{V} \hat{\mathbf{x}}_{j}
$$

(where the sequence $\left\{\tilde{\mathbf{x}}_{j}\right\}$ is known). The reduced variables $\hat{\mathbf{x}}_{j}$ satisfy the system

$$
\mathcal{V}^{T} \mathcal{M V}\left(\frac{\hat{\mathbf{x}}_{j}-\hat{\mathbf{x}}_{j-1}}{\Delta t}\right)=\mathcal{V}^{T} \mathcal{A} \mathcal{V}\left(\frac{\hat{\mathbf{x}}_{j}+\hat{\mathbf{x}}_{j-1}}{2}\right)+\mathcal{V}^{T}\left(\frac{\mathbf{f}_{j}+\mathbf{f}_{j-1}}{2}\right)+\mathcal{V}^{T} \mathcal{A}\left(\frac{\tilde{\mathbf{x}}_{j}+\tilde{\mathbf{x}}_{j-1}}{2}\right)-\mathcal{V}^{T} \mathcal{M}\left(\frac{\tilde{\mathbf{x}}_{j}-\tilde{\mathbf{x}}_{j-1}}{\Delta t}\right)
$$

with

$$
\hat{\mathbf{x}}_{0}=\left(\mathcal{V}^{T} \mathcal{M V}\right)^{-1} \mathcal{V}^{T} \mathcal{M}\left(\mathbf{x}_{0}-\tilde{\mathbf{x}}_{0}\right)
$$

Theorem 2.3 is modified as follows.

Theorem 2.7. The norms for the error between the approximation $\mathbf{x}_{j}$ and the discrete solution from the reduced order model (2.28) satisfy

$$
\begin{array}{r}
\Delta t \sum_{j=0}^{n_{t}}\left\|\mathbf{x}_{j}-\tilde{\mathbf{x}}_{j}-\mathcal{V} \hat{\mathbf{x}}_{j}\right\|_{\mathcal{M}}^{2} \leq\left(\Delta t \sum_{j=0}^{n_{t}}\left\|\mathbf{x}_{j}-\tilde{\mathbf{x}}_{j}-\mathcal{V}\left(\mathcal{V}^{T} \mathcal{M} \mathcal{V}\right)^{-1} \mathcal{V}^{T} \mathcal{M}\left(\mathbf{x}_{j}-\tilde{\mathbf{x}}_{j}\right)\right\|_{\mathcal{M}}^{2}\right) \\
\times\left[1+2 \alpha^{2} T\left(\Delta t \sum_{j=0}^{n_{t}} \mathrm{e}^{2 \gamma j \Delta t}\right)\right],
\end{array}
$$

where the coefficient $\alpha$ is

$$
\alpha=\frac{1}{2}\left\|\mathcal{M}^{\frac{1}{2}}\left(\mathcal{M}-\frac{\Delta t}{2} \mathcal{M V}\left(\mathcal{V}^{T} \mathcal{M V}\right)^{-1} \mathcal{V}^{T} \mathcal{A}\right)^{-1} \mathcal{M V}\left(\mathcal{V}^{T} \mathcal{M V}\right)^{-1} \mathcal{V}^{T} \mathcal{A Z}\left(\mathcal{Z}^{T} \mathcal{M} \mathcal{Z}\right)^{-1} \mathcal{Z}^{T} \mathcal{M}^{\frac{1}{2}}\right\|_{2}
$$

and the coefficient $\gamma$ is

$$
\gamma=\left\|\mathcal{M}^{\frac{1}{2}}\left(\mathcal{M}-\frac{\Delta t}{2} \mathcal{M V}\left(\mathcal{V}^{T} \mathcal{M V}\right)^{-1} \mathcal{V}^{T} \mathcal{A}\right)^{-1} \mathcal{M V}\left(\mathcal{V}^{T} \mathcal{M V}\right)^{-1} \mathcal{V}^{T} \mathcal{A} \mathcal{M}^{-\frac{1}{2}}\right\|_{2} .
$$

Remark 2.8. When the time-step $\Delta t$ goes to 0 , the right hand side of (2.29) remains bounded as

$$
\alpha \rightarrow \frac{1}{2}\left\|\mathcal{M}^{\frac{1}{2}} \mathcal{V}\left(\mathcal{V}^{T} \mathcal{M V}\right)^{-1} \mathcal{V}^{T} \mathcal{A Z}\left(\mathcal{Z}^{T} \mathcal{M} \mathcal{Z}\right)^{-1} \mathcal{Z}^{T} \mathcal{M}^{\frac{1}{2}}\right\|_{2}
$$

and

$$
\Delta t \sum_{j=0}^{n_{t}} \mathrm{e}^{2 \gamma j \Delta t} \rightarrow \int_{0}^{T} \mathrm{e}^{2 \gamma_{*} t} \mathrm{~d} t \quad \text { with } \gamma_{*}=\left\|\mathcal{M}^{\frac{1}{2}} \mathcal{V}\left(\mathcal{V}^{T} \mathcal{M} \mathcal{V}\right)^{-1} \mathcal{V}^{T} \mathcal{A} \mathcal{M}^{-\frac{1}{2}}\right\|_{2}
$$


The proper orthogonal decomposition yields, from the matrix

$$
\hat{\mathbf{R}}=\sum_{j=0}^{n_{t}}\left(\mathbf{x}_{j}-\tilde{\mathbf{x}}_{j}\right)\left(\mathbf{x}_{j}-\tilde{\mathbf{x}}_{j}\right)^{T}
$$

a matrix $\mathcal{V}$ whose columns are eigenvectors for the pencil $(\mathcal{M} \hat{\mathbf{R}} \mathcal{M}, \mathcal{M})$. Denoting $\left(\lambda_{i}\right)_{1 \leq i \leq r}$ the non-zero eigenvalues of the pencil - ordered in a non-increasing fashion - the projection error for this choice of matrix $\mathcal{V}$ is

$$
\sum_{j=0}^{n_{t}}\left\|\mathbf{x}_{j}-\tilde{\mathbf{x}}_{j}-\mathcal{V}\left(\mathcal{V}^{T} \mathcal{M} \mathcal{V}\right)^{-1} \mathcal{V}^{T} \mathcal{M}\left(\mathbf{x}_{j}-\tilde{\mathbf{x}}_{j}\right)\right\|_{\mathcal{M}}^{2}=\sum_{i=k+1}^{r} \lambda_{i} .
$$

The eigenvalues $\left(\lambda_{i}\right)_{1 \leq i \leq r}$ and the matrix $\mathcal{V}$ may also be computed via the eigendecomposition of

$$
\left[\mathbf{x}_{0}-\tilde{\mathbf{x}}_{0}, \ldots, \mathbf{x}_{n_{t}}-\tilde{\mathbf{x}}_{n_{t}}\right]^{T} \mathcal{M}\left[\mathbf{x}_{0}-\tilde{\mathbf{x}}_{0}, \ldots, \mathbf{x}_{n_{t}}-\tilde{\mathbf{x}}_{n_{t}}\right] .
$$

Results for the generalized first-order dynamical system (2.22) will be carefully used in the following section to analyze structure-preserving reduced-order models for the semi-discrete wave equation.

\section{ERRor EStimates FOR REDUCED-ORDER MODELS OF THE SEMI-DiSCRETE WAVE EQUATION}

\subsection{Error estimates for structure-preserving reduced-order models}

In this section, the semi-discrete wave equation is considered,

$$
\mathbf{M} \frac{\mathrm{d}^{2} \mathbf{u}}{\mathrm{d} t^{2}}(t)+\mathbf{D} \frac{\mathrm{d} \mathbf{u}}{\mathrm{d} t}(t)+\mathbf{K u}(t)=\mathbf{b}(t)
$$

where $\mathbf{M}$ is a symmetric positive definite matrix. For example, in structural dynamics, $\mathbf{M}$ is the mass matrix, $\mathbf{D}$ the damping matrix, and $\mathbf{K}$ the stiffness matrix. The objective is to analyze error estimates for structurepreserving reduced-order models of the form

$$
\hat{\mathbf{V}}^{T} \mathbf{M} \hat{\mathbf{V}} \frac{\mathrm{d}^{2} \mathbf{u}_{r}}{\mathrm{~d} t^{2}}(t)+\hat{\mathbf{V}}^{T} \mathbf{D} \hat{\mathbf{V}} \frac{\mathrm{d} \mathbf{u}_{r}}{\mathrm{~d} t}(t)+\hat{\mathbf{V}}^{T} \mathbf{K} \hat{\mathbf{V}} \mathbf{u}_{r}(t)=\mathbf{g}(t)
$$

where the columns of $\hat{\mathbf{V}}$ span the subspace of interest and $\mathbf{g}$ contains the reduced source terms.

The semi-discrete wave equation (3.1) can be reformulated as a first-order dynamical system,

$$
\left[\begin{array}{cc}
\mathbf{M} & \mathbf{0} \\
\mathbf{0} & \frac{1}{\tau^{2}} \mathbf{M}
\end{array}\right] \frac{\mathrm{d}}{\mathrm{d} t}\left(\left[\begin{array}{l}
\mathbf{v} \\
\mathbf{u}
\end{array}\right]\right)=\left[\begin{array}{cc}
-\mathbf{D} & -\mathbf{K} \\
\frac{1}{\tau^{2}} \mathbf{M} & \mathbf{0}
\end{array}\right]\left[\begin{array}{l}
\mathbf{v} \\
\mathbf{u}
\end{array}\right]+\left[\begin{array}{l}
\mathbf{b} \\
\mathbf{0}
\end{array}\right]
$$

with the initial condition

$$
\left[\begin{array}{l}
\mathbf{v} \\
\mathbf{u}
\end{array}\right](0)=\left[\begin{array}{l}
\mathbf{v}_{0} \\
\mathbf{u}_{0}
\end{array}\right]
$$

where $\tau$ is a characteristic time for the problem of interest. The scaling by $\frac{1}{\tau^{2}}$ is simply introduced to match the units for energy-like quantities, i.e.

$$
\left[\begin{array}{l}
\mathbf{v} \\
\mathbf{u}
\end{array}\right]^{T}\left[\begin{array}{cc}
\mathbf{M} & \mathbf{0} \\
\mathbf{0} & \frac{1}{\tau^{2}} \mathbf{M}
\end{array}\right]\left[\begin{array}{l}
\mathbf{v} \\
\mathbf{u}
\end{array}\right]=\mathbf{v}^{T} \mathbf{M} \mathbf{v}+\frac{1}{\tau^{2}} \mathbf{u}^{T} \mathbf{M u}
$$

where $\mathbf{v}$ is a vector of velocities and $\mathbf{u}$ a vector of displacements. 
A reduced-order model is built for the approximation

$$
\left[\begin{array}{l}
\mathbf{v}_{\mathrm{ROM}}(t) \\
\mathbf{u}_{\mathrm{ROM}}(t)
\end{array}\right]=\left[\begin{array}{c}
\frac{\mathrm{d} \tilde{\mathbf{u}}}{\mathrm{d} t}(t) \\
\tilde{\mathbf{u}}(t)
\end{array}\right]+\left[\begin{array}{cc}
\hat{\mathbf{V}} & \mathbf{0} \\
\mathbf{0} & \hat{\mathbf{V}}
\end{array}\right]\left[\begin{array}{l}
\mathbf{v}_{r}(t) \\
\mathbf{u}_{r}(t)
\end{array}\right],
$$

where the function $\tilde{\mathbf{u}}$ is known a priori (for example, $\tilde{\mathbf{u}}(t)=\mathbf{u}_{0}+\mathbf{v}_{0} t$ ). The reduced variables $\mathbf{v}_{r}$ and $\mathbf{u}_{r}$ satisfy

$$
\begin{aligned}
{\left[\begin{array}{cc}
\hat{\mathbf{V}}^{T} \mathbf{M} \hat{\mathbf{V}} & \mathbf{0} \\
\mathbf{0} & \frac{1}{\tau^{2}} \hat{\mathbf{V}}^{T} \mathbf{M} \hat{\mathbf{V}}
\end{array}\right] \frac{\mathrm{d}}{\mathrm{d} t}\left(\left[\begin{array}{c}
\mathbf{v}_{r} \\
\mathbf{u}_{r}
\end{array}\right]\right)(t)=} & {\left[\begin{array}{cc}
-\hat{\mathbf{V}}^{T} \mathbf{D} \hat{\mathbf{V}} & -\hat{\mathbf{V}}^{T} \mathbf{K} \hat{\mathbf{V}} \\
\frac{1}{\tau^{2}} \hat{\mathbf{V}}^{T} \mathbf{M} \hat{\mathbf{V}} & \mathbf{0}
\end{array}\right]\left[\begin{array}{c}
\mathbf{v}_{r}(t) \\
\mathbf{u}_{r}(t)
\end{array}\right]+\left[\begin{array}{c}
\hat{\mathbf{V}}^{T} \mathbf{b}(t) \\
\mathbf{0}
\end{array}\right] } \\
& +\left[\begin{array}{cc}
-\hat{\mathbf{V}}^{T} \mathbf{D} & -\hat{\mathbf{V}}^{T} \mathbf{K} \\
\frac{1}{\tau^{2}} \hat{\mathbf{V}}^{T} \mathbf{M} & \mathbf{0}
\end{array}\right]\left[\begin{array}{c}
\frac{\mathrm{d} \tilde{\mathbf{u}}}{\mathrm{d} t}(t) \\
\tilde{\mathbf{u}}(t)
\end{array}\right]-\left[\begin{array}{cc}
\hat{\mathbf{V}}^{T} \mathbf{M} & \mathbf{0} \\
\mathbf{0} & \frac{1}{\tau^{2}} \hat{\mathbf{V}}^{T} \mathbf{M}
\end{array}\right]\left[\begin{array}{c}
\frac{\mathrm{d}^{2} \tilde{u}}{\mathrm{~d} t^{2}}(t) \\
\frac{\mathrm{du}}{\mathrm{d} t}(t)
\end{array}\right] .
\end{aligned}
$$

The second equation implies that the approximation $\mathbf{v}_{\text {ROM }}$ satisfies

$$
\mathbf{v}_{\mathrm{ROM}}(t)=\frac{\mathrm{d} \mathbf{u}_{\mathrm{ROM}}}{\mathrm{d} t}(t),
$$

i.e. this structure is preserved for the reduced-order model ${ }^{5}$. The corresponding second-order equation is

$$
\hat{\mathbf{V}}^{T} \mathbf{M} \hat{\mathbf{V}} \frac{\mathrm{d}^{2} \mathbf{u}_{r}}{\mathrm{~d} t^{2}}(t)+\hat{\mathbf{V}}^{T} \mathbf{D} \hat{\mathbf{V}} \frac{\mathrm{d} \mathbf{u}_{r}}{\mathrm{~d} t}(t)+\hat{\mathbf{V}}^{T} \mathbf{K} \hat{\mathbf{V}} \mathbf{u}_{r}(t)=\hat{\mathbf{V}}^{T} \mathbf{b}(t)-\hat{\mathbf{V}}^{T} \mathbf{M} \frac{\mathrm{d}^{2} \tilde{\mathbf{u}}}{\mathrm{d} t^{2}}(t)-\hat{\mathbf{V}}^{T} \mathbf{D} \frac{\mathrm{d} \tilde{\mathbf{u}}}{\mathrm{d} t}(t)-\hat{\mathbf{V}}^{T} \mathbf{K} \tilde{\mathbf{u}}(t),
$$

indicating that the second-order structure and the symmetry of matrices (like $\mathbf{M}$ and $\hat{\mathbf{V}}^{T} \mathbf{M} \hat{\mathbf{V}}$ ) are preserved. The right hand side is modified to reflect the incremental representation (3.5). The initial conditions for the reduced variables $\mathbf{u}_{r}$ and $\mathbf{v}_{r}$ are

$$
\mathbf{u}_{r}(0)=\left(\hat{\mathbf{V}}^{T} \mathbf{M} \hat{\mathbf{V}}\right)^{-1} \hat{\mathbf{V}}^{T} \mathbf{M}\left(\mathbf{u}_{0}-\tilde{\mathbf{u}}(0)\right) \quad \text { and } \quad \mathbf{v}_{r}(0)=\left(\hat{\mathbf{V}}^{T} \mathbf{M} \hat{\mathbf{V}}\right)^{-1} \hat{\mathbf{V}}^{T} \mathbf{M}\left(\mathbf{v}_{0}-\frac{\mathrm{d} \tilde{\mathbf{u}}}{\mathrm{d} t}(0)\right) .
$$

To apply the previous results, the following identifications are made

$$
\mathbf{x} \leftrightarrow\left[\begin{array}{l}
\mathbf{v} \\
\mathbf{u}
\end{array}\right], \quad \mathcal{V} \leftrightarrow\left[\begin{array}{ll}
\hat{\mathbf{V}} & \mathbf{0} \\
\mathbf{0} & \hat{\mathbf{V}}
\end{array}\right], \quad \mathcal{M} \leftrightarrow\left[\begin{array}{cc}
\mathbf{M} & \mathbf{0} \\
\mathbf{0} & \frac{1}{\tau^{2}} \mathbf{M}
\end{array}\right], \quad \mathcal{A} \leftrightarrow\left[\begin{array}{cc}
-\mathbf{D} & -\mathbf{K} \\
\frac{1}{\tau^{2}} \mathbf{M} & \mathbf{0}
\end{array}\right] .
$$

Consequently, the errors satisfy the relationships

$$
\left\|\mathcal{E}_{\mathrm{ROM}}(t)\right\|_{\mathcal{M}}^{2} \leftrightarrow \frac{1}{\tau^{2}}\left\|\mathbf{u}(t)-\tilde{\mathbf{u}}(t)-\hat{\mathbf{V}} \mathbf{u}_{r}(t)\right\|_{\mathbf{M}}^{2}+\left\|\frac{\mathrm{d} \mathbf{u}}{\mathrm{d} t}(t)-\frac{\mathrm{d} \tilde{\mathbf{u}}}{\mathrm{d} t}(t)-\hat{\mathbf{V}} \frac{\mathrm{d} \mathbf{u}_{r}}{\mathrm{~d} t}(t)\right\|_{\mathbf{M}}^{2}
$$

and

$$
\begin{aligned}
\| \mathbf{x}(t)-\tilde{\mathbf{x}}(t)-\mathcal{V}\left(\mathcal{V}^{T} \mathcal{M V}\right)^{-1} & \mathcal{V}^{T} \mathcal{M}(\mathbf{x}(t)-\tilde{\mathbf{x}}(t)) \|_{\mathcal{M}}^{2} \\
\leftrightarrow \frac{1}{\tau^{2}} \| \mathbf{u}(t)-\tilde{\mathbf{u}}(t) & -\hat{\mathbf{V}}\left(\hat{\mathbf{V}}^{T} \mathbf{M} \hat{\mathbf{V}}\right)^{-1} \hat{\mathbf{V}}^{T} \mathbf{M}(\mathbf{u}(t)-\tilde{\mathbf{u}}(t)) \|_{\mathbf{M}}^{2} \\
& +\left\|\frac{\mathrm{d} \mathbf{u}}{\mathrm{d} t}(t)-\frac{\mathrm{d} \tilde{\mathbf{u}}}{\mathrm{d} t}(t)-\hat{\mathbf{V}}\left(\hat{\mathbf{V}}^{T} \mathbf{M} \hat{\mathbf{V}}\right)^{-1} \hat{\mathbf{V}}^{T} \mathbf{M}\left(\frac{\mathrm{d} \mathbf{u}}{\mathrm{d} t}(t)-\frac{\mathrm{d} \tilde{\mathbf{u}}}{\mathrm{d} t}(t)\right)\right\|_{\mathbf{M}}^{2}
\end{aligned}
$$

When decomposing $\mathbb{R}^{2 n}$ as

$$
\mathbb{R}^{2 n}=\operatorname{span} \mathcal{V} \oplus^{\perp_{\mathcal{M}}} \operatorname{span} \mathcal{Z}=\operatorname{span}\left[\begin{array}{cc}
\hat{\mathbf{V}} & \mathbf{0} \\
\mathbf{0} & \hat{\mathbf{V}}
\end{array}\right] \oplus^{\perp_{\mathcal{M}}} \operatorname{span} \mathcal{Z}
$$

Theorem 2.1 is modified as follows.

\footnotetext{
${ }^{5}$ This feature results also from the choice of $\left[\begin{array}{c}\frac{\mathrm{d} \tilde{\mathbf{u}}}{\mathrm{d} t}(t) \\ \tilde{\mathbf{u}}(t)\end{array}\right]$ in the incremental representation (3.5).
} 
Theorem 3.1. The error between the solution, $\mathbf{u}$, (3.1) and the approximation, $\mathbf{u}_{\mathrm{ROM}}=\tilde{\mathbf{u}}+\hat{\mathbf{V}} \mathbf{u}_{r}$, (3.7) satisfies

$$
\begin{aligned}
\int_{0}^{T} \frac{\left\|\mathbf{u}(t)-\mathbf{u}_{\mathrm{ROM}}(t)\right\|_{\mathbf{M}}^{2}}{\tau^{2}}+\| & \frac{\mathrm{d} \mathbf{u}}{\mathrm{d} t}(t)-\frac{\mathrm{d} \mathbf{u}_{\mathrm{ROM}}}{\mathrm{d} t}(t) \|_{\mathbf{M}}^{2} \mathrm{~d} t \\
\leq(1+ & \left.\left\|F\left(T,\left(\mathcal{V}^{T} \mathcal{M V}\right)^{-1} \mathcal{V}^{T} \mathcal{A} \mathcal{V}\right)\right\|_{\mathcal{V}^{T} \mathcal{M} \mathcal{V}}^{2}\left\|\mathcal{V}^{T} \mathcal{A} \mathcal{Z}\left(\mathcal{Z}^{T} \mathcal{M} \mathcal{Z}\right)^{-1} \mathcal{Z}^{T} \mathcal{M}^{\frac{1}{2}}\right\|_{\left(\mathcal{V}^{T} \mathcal{M V}\right)^{-1}}^{2}\right) \\
& \times\left(\int_{0}^{T} \frac{1}{\tau^{2}}\left\|\mathbf{u}(t)-\tilde{\mathbf{u}}(t)-\hat{\mathbf{V}}\left(\hat{\mathbf{V}}^{T} \mathbf{M} \hat{\mathbf{V}}\right)^{-1} \hat{\mathbf{V}}^{T} \mathbf{M}(\mathbf{u}(t)-\tilde{\mathbf{u}}(t))\right\|_{\mathbf{M}}^{2} \mathrm{~d} t\right. \\
& \left.\quad+\int_{0}^{T}\left\|\frac{\mathrm{d} \mathbf{u}}{\mathrm{d} t}(t)-\frac{\mathrm{d} \tilde{\mathbf{u}}}{\mathrm{d} t}(t)-\hat{\mathbf{V}}\left(\hat{\mathbf{V}}^{T} \mathbf{M} \hat{\mathbf{V}}\right)^{-1} \hat{\mathbf{V}}^{T} \mathbf{M}\left(\frac{\mathrm{d} \mathbf{u}}{\mathrm{d} t}(t)-\frac{\mathrm{d} \tilde{\mathbf{u}}}{\mathrm{d} t}(t)\right)\right\|_{\mathbf{M}}^{2} \mathrm{~d} t\right),
\end{aligned}
$$

where the constant $\left\|F\left(T,\left(\mathcal{V}^{T} \mathcal{M V}\right)^{-1} \mathcal{V}^{T} \mathcal{A} \mathcal{V}\right)\right\|_{\mathcal{V}^{T} \mathcal{M V}}$ is defined by (2.11).

Assuming that the entire continuous trajectory $\mathbf{u}(t)$ is available on the interval $[0 ; T]$, results in Section 2.2 indicate that eigenspaces for the pencil

$$
\left(\left[\begin{array}{cc}
\int_{0}^{T} \mathbf{M}\left(\frac{\mathrm{d} \mathbf{u}}{\mathrm{d} t}(t)-\frac{\mathrm{d} \tilde{\mathbf{u}}}{\mathrm{d} t}(t)\right)\left(\frac{\mathrm{d} \mathbf{u}}{\mathrm{d} t}(t)-\frac{\mathrm{d} \tilde{\mathbf{u}}}{\mathrm{d} t}(t)\right)^{T} \mathbf{M} \mathrm{d} t & \frac{1}{\tau^{2}} \int_{0}^{T} \mathbf{M}\left(\frac{\mathrm{d} \mathbf{u}}{\mathrm{d} t}(t)-\frac{\mathrm{d} \tilde{\mathbf{u}}}{\mathrm{d} t}(t)\right)(\mathbf{u}(t)-\tilde{\mathbf{u}}(t))^{T} \mathbf{M} \mathrm{d} t \\
\frac{1}{\tau^{2}} \int_{0}^{T} \mathbf{M}(\mathbf{u}(t)-\tilde{\mathbf{u}}(t))\left(\frac{\mathrm{d} \mathbf{u}}{\mathrm{d} t}(t)-\frac{\mathrm{d} \tilde{\mathbf{u}}}{\mathrm{d} t}(t)\right)^{T} \mathbf{M} \mathrm{d} t & \frac{1}{\tau^{4}} \int_{0}^{T} \mathbf{M}(\mathbf{u}(t)-\tilde{\mathbf{u}}(t))(\mathbf{u}(t)-\tilde{\mathbf{u}}(t))^{T} \mathbf{M} \mathrm{d} t
\end{array}\right],\left[\begin{array}{cc}
\mathbf{M} & \mathbf{0} \\
\mathbf{0} & \frac{1}{\tau^{2}} \mathbf{M}
\end{array}\right]\right)
$$

have good approximation properties. Unfortunately, these resulting eigenspaces are not guaranteed to preserve the structure of the semi-discrete wave equation. If, instead, the subspace spanned by $\hat{\mathbf{V}}$ is selected to minimize the projection error in (3.9), then the matrix $\hat{\mathbf{V}}$ satisfies

$$
\begin{aligned}
\hat{\mathbf{V}}=\arg \max _{\operatorname{rank} \mathbf{V}=k} \int_{0}^{T} \operatorname{tr}\left(\mathbf{M V}\left(\mathbf{V}^{T} \mathbf{M V}\right)^{-1} \mathbf{V}^{T} \mathbf{M}\left(\frac{\mathrm{d} \mathbf{u}}{\mathrm{d} t}(t)-\frac{\mathrm{d} \tilde{\mathbf{u}}}{\mathrm{d} t}(t)\right)\left(\frac{\mathrm{d} \mathbf{u}}{\mathrm{d} t}(t)-\frac{\mathrm{d} \tilde{\mathbf{u}}}{\mathrm{d} t}(t)\right)^{T}\right) \\
+\frac{1}{\tau^{2}} \operatorname{tr}\left(\mathbf{M V}\left(\mathbf{V}^{T} \mathbf{M V}\right)^{-1} \mathbf{V}^{T} \mathbf{M}(\mathbf{u}(t)-\tilde{\mathbf{u}}(t))(\mathbf{u}(t)-\tilde{\mathbf{u}}(t))^{T}\right) \mathrm{d} t
\end{aligned}
$$

Following the derivation in the appendix, a minimizing basis $\hat{\mathbf{V}}$ will span a stable subspace for the pencil (MRM, M), where the matrix $\mathbf{R}$ is defined as

$$
\mathbf{R}=\int_{0}^{T}\left(\frac{\mathrm{d} \mathbf{u}}{\mathrm{d} t}(t)-\frac{\mathrm{d} \tilde{\mathbf{u}}}{\mathrm{d} t}(t)\right)\left(\frac{\mathrm{d} \mathbf{u}}{\mathrm{d} t}(t)-\frac{\mathrm{d} \tilde{\mathbf{u}}}{\mathrm{d} t}(t)\right)^{T}+\frac{1}{\tau^{2}}(\mathbf{u}(t)-\tilde{\mathbf{u}}(t))(\mathbf{u}(t)-\tilde{\mathbf{u}}(t))^{T} \mathrm{~d} t .
$$

Denoting $\left(\lambda_{i}^{\infty}\right)_{1 \leq i \leq r}$ the non-zero eigenvalues of the pencil (MRM, M) - ordered in a non-increasing fashion the projection error for the minimization problem becomes

$$
\begin{aligned}
\int_{0}^{T} \frac{1}{\tau^{2}} \| \mathbf{u}(t)-\tilde{\mathbf{u}}(t)-\hat{\mathbf{V}} & \left(\hat{\mathbf{V}}^{T} \mathbf{M} \hat{\mathbf{V}}\right)^{-1} \hat{\mathbf{V}}^{T} \mathbf{M}(\mathbf{u}(t)-\tilde{\mathbf{u}}(t)) \|_{\mathbf{M}}^{2} \mathrm{~d} t \\
& +\int_{0}^{T}\left\|\frac{\mathrm{d} \mathbf{u}}{\mathrm{d} t}(t)-\frac{\mathrm{d} \tilde{\mathbf{u}}}{\mathrm{d} t}(t)-\hat{\mathbf{V}}\left(\hat{\mathbf{V}}^{T} \mathbf{M} \hat{\mathbf{V}}\right)^{-1} \hat{\mathbf{V}}^{T} \mathbf{M}\left(\frac{\mathrm{d} \mathbf{u}}{\mathrm{d} t}(t)-\frac{\mathrm{d} \tilde{\mathbf{u}}}{\mathrm{d} t}(t)\right)\right\|_{\mathbf{M}}^{2} \mathrm{~d} t=\sum_{i=k+1}^{r} \lambda_{i}^{\infty}
\end{aligned}
$$

Remark 3.2. The error bound (3.9) could be written for the quantity

$$
\int_{0}^{T}\left\|\mathbf{u}(t)-\mathbf{u}_{\mathrm{ROM}}(t)\right\|_{\mathbf{K}}^{2}+\left\|\frac{\mathrm{d} \mathbf{u}}{\mathrm{d} t}(t)-\frac{\mathrm{d} \mathbf{u}_{\mathrm{ROM}}}{\mathrm{d} t}(t)\right\|_{\mathbf{M}}^{2} \mathrm{~d} t
$$


by using the correspondance

$$
\mathcal{M} \leftrightarrow\left[\begin{array}{cc}
\mathbf{M} & \mathbf{0} \\
\mathbf{0} & \mathbf{K}
\end{array}\right] \quad \text { and } \quad \mathcal{A} \leftrightarrow\left[\begin{array}{cc}
-\mathbf{D} & -\mathbf{K} \\
\mathbf{K} & \mathbf{0}
\end{array}\right] .
$$

In this case, the minimization problem for the projection error becomes

$$
\begin{aligned}
\hat{\mathbf{V}}=\arg \min _{\operatorname{rank} \mathbf{V}=k} \int_{0}^{T}\left\|\mathbf{u}(t)-\tilde{\mathbf{u}}(t)-\mathbf{V}\left(\mathbf{V}^{T} \mathbf{K V}\right)^{-1} \mathbf{V}^{T} \mathbf{K}(\mathbf{u}(t)-\tilde{\mathbf{u}}(t))\right\|_{\mathbf{K}}^{2} & \\
& +\left\|\frac{\mathrm{d} \mathbf{u}}{\mathrm{d} t}(t)-\frac{\mathrm{d} \tilde{\mathbf{u}}}{\mathrm{d} t}(t)-\mathbf{V}\left(\mathbf{V}^{T} \mathbf{M V}\right)^{-1} \mathbf{V}^{T} \mathbf{M}\left(\frac{\mathrm{d} \mathbf{u}}{\mathrm{d} t}(t)-\frac{\mathrm{d} \tilde{\mathbf{u}}}{\mathrm{d} t}(t)\right)\right\|_{\mathbf{M}}^{2} \mathrm{~d} t .
\end{aligned}
$$

However the Euler-Lagrange equation for this minimization problem yields a matrix equation whose solution remains challenging.

Next consider the Newmark scheme $\left(\gamma=\frac{1}{2}, \beta=\frac{1}{4}\right)$ applied to (3.1),

$$
\left\{\begin{array}{l}
\mathbf{M} \mathbf{a}_{j+1}+\mathbf{D} \mathbf{v}_{j+1}+\mathbf{K} \mathbf{u}_{j+1}=\mathbf{b}_{j+1} \\
\mathbf{v}_{j+1}=\mathbf{v}_{j}+\frac{\Delta t}{2}\left[\mathbf{a}_{j}+\mathbf{a}_{j+1}\right] \\
\mathbf{u}_{j+1}=\mathbf{u}_{j}+\frac{\Delta t}{2}\left[\mathbf{v}_{j}+\mathbf{v}_{j+1}\right]
\end{array}\right.
$$

where $\left(\mathbf{a}_{j}, \mathbf{v}_{j}, \mathbf{u}_{j}\right)$ approximates $\left(\frac{\mathrm{d}^{2} \mathbf{u}}{\mathrm{d} t^{2}}(j \Delta t), \frac{\mathrm{d} \mathbf{u}}{\mathrm{d} t}(j \Delta t), \mathbf{u}(j \Delta t)\right)$. This scheme, also known as the average acceleration method, matches the system obtained from trapezoidal time integration on (3.3),

$$
\left\{\begin{array}{l}
\mathbf{M}\left(\frac{\mathbf{v}_{j}-\mathbf{v}_{j-1}}{\Delta t}\right)=-\mathbf{D}\left(\frac{\mathbf{v}_{j}+\mathbf{v}_{j-1}}{2}\right)-\mathbf{K}\left(\frac{\mathbf{u}_{j}+\mathbf{u}_{j-1}}{2}\right)+\frac{\mathbf{b}_{j}+\mathbf{b}_{j-1}}{2} \\
\frac{1}{\tau^{2}} \mathbf{M}\left(\frac{\mathbf{u}_{j}-\mathbf{u}_{j-1}}{\Delta t}\right)=\frac{1}{\tau^{2}} \mathbf{M}\left(\frac{\mathbf{v}_{j}+\mathbf{v}_{j-1}}{2}\right)
\end{array}\right.
$$

(see [17], p. 495). Hence the analysis from Section 2.2 will give error estimates when applying the Newmark scheme to a reduced-order model.

An approximation is constructed of the form

$$
\left[\begin{array}{l}
\mathbf{v}_{j} \\
\mathbf{u}_{j}
\end{array}\right] \approx\left[\begin{array}{c}
\tilde{\mathbf{v}}_{j} \\
\tilde{\mathbf{u}}_{j}
\end{array}\right]+\left[\begin{array}{c}
\hat{\mathbf{V}} \hat{\mathbf{v}}_{j} \\
\hat{\mathbf{V}} \hat{\mathbf{u}}_{j}
\end{array}\right]
$$

where the sequences $\left\{\tilde{\mathbf{u}}_{j}\right\}$ and $\left\{\tilde{\mathbf{v}}_{j}\right\}$ are selected appropriately to preserve the structure of the wave equation. Given an a priori known sequence $\left\{\tilde{\mathbf{a}}_{j}\right\}$, the sequences $\left\{\tilde{\mathbf{u}}_{j}\right\}$ and $\left\{\tilde{\mathbf{v}}_{j}\right\}$ are required to satisfy the steps for the Newmark scheme, i.e.

$$
\left\{\begin{array}{l}
\tilde{\mathbf{v}}_{j+1}=\tilde{\mathbf{v}}_{j}+\frac{\Delta t}{2}\left[\tilde{\mathbf{a}}_{j}+\tilde{\mathbf{a}}_{j+1}\right] \\
\tilde{\mathbf{u}}_{j+1}=\tilde{\mathbf{u}}_{j}+\frac{\Delta t}{2}\left[\tilde{\mathbf{v}}_{j}+\tilde{\mathbf{v}}_{j+1}\right]
\end{array}\right.
$$

The reduced variables $\left\{\hat{\mathbf{u}}_{j}\right\}$ and $\left\{\hat{\mathbf{v}}_{j}\right\}$ satisfy the system of equations

$$
\begin{aligned}
& \frac{1}{\Delta t}\left[\begin{array}{cc}
\hat{\mathbf{V}}^{T} \mathbf{M} \hat{\mathbf{V}} & \mathbf{0} \\
\mathbf{0} & \frac{1}{\tau^{2}} \hat{\mathbf{V}}^{T} \mathbf{M} \hat{\mathbf{V}}
\end{array}\right]\left[\begin{array}{c}
\hat{\mathbf{V}}_{j}-\hat{\mathbf{V}}_{j-1} \\
\hat{\mathbf{u}}_{j}-\hat{\mathbf{u}}_{j-1}
\end{array}\right]=\frac{1}{2}\left[\begin{array}{cc}
-\hat{\mathbf{V}}^{T} \mathbf{D} \hat{\mathbf{V}} & -\hat{\mathbf{V}}^{T} \mathbf{K} \hat{\mathbf{V}} \\
\frac{1}{\tau^{2}} \hat{\mathbf{V}}^{T} \mathbf{M} \hat{\mathbf{V}} & \mathbf{0}
\end{array}\right]\left[\begin{array}{c}
\hat{\mathbf{V}}_{j}+\hat{\mathbf{V}}_{j-1} \\
\hat{\mathbf{u}}_{j}+\hat{\mathbf{u}}_{j-1}
\end{array}\right] \\
& -\frac{1}{\Delta t}\left[\begin{array}{cc}
\hat{\mathbf{V}}^{T} \mathbf{M} & \mathbf{0} \\
\mathbf{0} & \frac{1}{\tau^{2}} \hat{\mathbf{V}}^{T} \mathbf{M}
\end{array}\right]\left[\begin{array}{c}
\tilde{\mathbf{V}}_{j}-\tilde{\mathbf{v}}_{j-1} \\
\tilde{\mathbf{u}}_{j}-\tilde{\mathbf{u}}_{j-1}
\end{array}\right]+\frac{1}{2}\left[\begin{array}{cc}
-\hat{\mathbf{V}}^{T} \mathbf{D} & -\hat{\mathbf{V}}^{T} \mathbf{K} \\
\frac{1}{\tau^{2}} \hat{\mathbf{V}}^{T} \mathbf{M} & \mathbf{0}
\end{array}\right]\left[\begin{array}{c}
\tilde{\mathbf{v}}_{j}+\tilde{\mathbf{v}}_{j-1} \\
\tilde{\mathbf{u}}_{j}+\tilde{\mathbf{u}}_{j-1}
\end{array}\right] \\
& +\frac{1}{2}\left[\begin{array}{c}
\hat{\mathbf{V}}^{T} \mathbf{b}_{j}+\hat{\mathbf{V}}^{T} \mathbf{b}_{j-1} \\
\mathbf{0}
\end{array}\right] \text {. }
\end{aligned}
$$


The second equation implies that the sequences $\left\{\hat{\mathbf{u}}_{j}\right\}$ and $\left\{\hat{\mathbf{v}}_{j}\right\}$ satisfy

$$
\hat{\mathbf{V}}^{T} \mathbf{M} \hat{\mathbf{V}}\left(\hat{\mathbf{u}}_{j}-\hat{\mathbf{u}}_{j-1}\right)=\frac{\Delta t}{2} \hat{\mathbf{V}}^{T} \mathbf{M} \hat{\mathbf{V}}\left(\hat{\mathbf{v}}_{j}+\hat{\mathbf{v}}_{j-1}\right)
$$

because of assumption (3.11). The initial conditions are

$$
\hat{\mathbf{v}}_{0}=\left(\hat{\mathbf{V}}^{T} \mathbf{M} \hat{\mathbf{V}}\right)^{-1} \hat{\mathbf{V}}^{T} \mathbf{M}\left(\mathbf{v}_{0}-\tilde{\mathbf{v}}_{0}\right) \quad \text { and } \quad \hat{\mathbf{u}}_{0}=\left(\hat{\mathbf{V}}^{T} \mathbf{M} \hat{\mathbf{V}}\right)^{-1} \hat{\mathbf{V}}^{T} \mathbf{M}\left(\mathbf{u}_{0}-\tilde{\mathbf{u}}_{0}\right) .
$$

This reduced-order model is obtained by applying the trapezoidal time integration on (3.6). The relationship between the trapezoidal time integration scheme and the average acceleration method implies that the model (3.12) matches the scheme obtained with the average acceleration method on (3.7).

Theorem 3.3. The norms for the error between the approximation $\left(\mathbf{u}_{j}, \mathbf{v}_{j}\right)$ and the discrete solution from the reduced order model (3.12) satisfy

$$
\begin{aligned}
\sum_{j=0}^{n_{t}} \frac{1}{\tau^{2}}\left\|\mathbf{u}_{j}-\tilde{\mathbf{u}}_{j}-\hat{\mathbf{V}} \hat{\mathbf{u}}_{j}\right\|_{\mathbf{M}}^{2} & +\left\|\mathbf{v}_{j}-\tilde{\mathbf{v}}_{j}-\hat{\mathbf{V}} \hat{\mathbf{v}}_{j}\right\|_{\mathbf{M}}^{2} \leq\left[1+2 \alpha^{2} T\left(\Delta t \sum_{j=0}^{n_{t}} \mathrm{e}^{2 \gamma j \Delta t}\right)\right] \\
& \times\left(\frac{1}{\tau^{2}} \sum_{j=0}^{n_{t}}\left\|\mathbf{u}_{j}-\tilde{\mathbf{u}}_{j}-\hat{\mathbf{V}}\left(\hat{\mathbf{V}}^{T} \mathbf{M} \hat{\mathbf{V}}\right)^{-1} \hat{\mathbf{V}}^{T} \mathbf{M}\left(\mathbf{u}_{j}-\tilde{\mathbf{u}}_{j}\right)\right\|_{\mathbf{M}}^{2}\right. \\
& \left.+\sum_{j=0}^{n_{t}}\left\|\mathbf{v}_{j}-\tilde{\mathbf{v}}_{j}-\hat{\mathbf{V}}\left(\hat{\mathbf{V}}^{T} \mathbf{M} \hat{\mathbf{V}}\right)^{-1} \hat{\mathbf{V}}^{T} \mathbf{M}\left(\mathbf{v}_{j}-\tilde{\mathbf{v}}_{j}\right)\right\|_{\mathbf{M}}^{2}\right)
\end{aligned}
$$

where the constants $\alpha$ and $\gamma$ are defined in Theorem 2.7 with the identifications (3.8).

Derivations in the appendix indicate that the proper orthogonal decomposition generates, from the matrix

$$
\hat{\mathbf{R}}=\sum_{j=0}^{n_{t}}\left(\mathbf{v}_{j}-\tilde{\mathbf{v}}_{j}\right)\left(\mathbf{v}_{j}-\tilde{\mathbf{v}}_{j}\right)^{T}+\frac{1}{\tau^{2}}\left(\mathbf{u}_{j}-\tilde{\mathbf{u}}_{j}\right)\left(\mathbf{u}_{j}-\tilde{\mathbf{u}}_{j}\right)^{T}
$$

a matrix $\hat{\mathbf{V}}$ whose columns are the dominant eigenvectors for the pencil ( $\mathbf{M} \hat{\mathbf{R}} \mathbf{M}, \mathbf{M})$. Denoting $\left(\lambda_{i}\right)_{1 \leq i \leq r}$ the non-zero eigenvalues of the pencil - ordered in a non-increasing fashion - the projection error for this choice of matrix $\hat{\mathbf{V}}$ is

$$
\begin{aligned}
\sum_{j=0}^{n_{t}}\left\|\mathbf{v}_{j}-\tilde{\mathbf{v}}_{j}-\hat{\mathbf{V}}\left(\hat{\mathbf{V}}^{T} \mathbf{M} \hat{\mathbf{V}}\right)^{-1} \hat{\mathbf{V}}^{T} \mathbf{M}\left(\mathbf{v}_{j}-\tilde{\mathbf{v}}_{j}\right)\right\|_{\mathbf{M}}^{2} & \\
& +\frac{1}{\tau^{2}} \sum_{j=0}^{n_{t}}\left\|\mathbf{u}_{j}-\tilde{\mathbf{u}}_{j}-\hat{\mathbf{V}}\left(\hat{\mathbf{V}}^{T} \mathbf{M} \hat{\mathbf{V}}\right)^{-1} \hat{\mathbf{V}}^{T} \mathbf{M}\left(\mathbf{u}_{j}-\tilde{\mathbf{u}}_{j}\right)\right\|_{\mathbf{M}}^{2}=\sum_{i=k+1}^{r} \lambda_{i} .
\end{aligned}
$$

In practice, the eigenvalues $\left(\lambda_{i}\right)_{1 \leq i \leq r}$ are computed through the eigenvalue decomposition of the correlation matrix

$$
\left[\begin{array}{c}
\left(\mathbf{v}_{0}-\tilde{\mathbf{v}}_{0}\right)^{T} \\
\vdots \\
\left(\mathbf{v}_{n_{t}}-\tilde{\mathbf{v}}_{n_{t}}\right)^{T} \\
\frac{1}{\tau}\left(\mathbf{u}_{0}-\tilde{\mathbf{u}}_{0}\right)^{T} \\
\vdots \\
\frac{1}{\tau}\left(\mathbf{u}_{n_{t}}-\tilde{\mathbf{u}}_{n_{t}}\right)^{T}
\end{array}\right] \mathbf{M}\left[\left(\mathbf{v}_{0}-\tilde{\mathbf{v}}_{0}\right) \ldots\left(\mathbf{v}_{n_{t}}-\tilde{\mathbf{v}}_{n_{t}}\right) \frac{1}{\tau}\left(\mathbf{u}_{0}-\tilde{\mathbf{u}}_{0}\right) \ldots \frac{1}{\tau}\left(\mathbf{u}_{n_{t}}-\tilde{\mathbf{u}}_{n_{t}}\right)\right] \text {. }
$$

Theorem 3.3 justifies the heuristic argument that the error when approximating the full state variables is controlled by the sum of neglected singular values, obtained from the proper orthogonal decomposition. 


\subsection{Snapshots choice}

Theorem 3.3 suggests to use a set of snapshots composed of velocities and displacements. This choice is reminiscent of the one made by Kunisch and Volkwein [21]. In the following, different snapshot matrices are discussed and related to the error estimate (3.13). It will be shown that the projection error is bounded for each choice of the snapshots in terms of the corresponding neglected eigenvalues.

Denoting by $\mathbb{U}, \mathbb{V}$, and $\mathbb{A}$ the snapshot matrices for the displacement, velocity, and acceleration, respectively,

$$
\mathbb{U}=\left[\mathbf{u}_{0}, \mathbf{u}_{1}, \ldots, \mathbf{u}_{n_{t}}\right], \mathbb{V}=\left[\mathbf{v}_{0}, \mathbf{v}_{1}, \ldots, \mathbf{v}_{n_{t}}\right], \mathbb{A}=\left[\mathbf{a}_{0}, \mathbf{a}_{1}, \ldots, \mathbf{a}_{n_{t}}\right],
$$

the average acceleration time-integration scheme enforces the identities

$$
\mathbb{V}=\mathbf{v}_{0}\left[\begin{array}{ll}
1 & \mathbf{1}^{T}
\end{array}\right]+\frac{\Delta t}{2} \mathbb{A}\left[\begin{array}{cc}
0 & \mathbf{1}^{T} \\
\mathbf{0} & \tilde{\mathbb{T}}
\end{array}\right] \quad \text { and } \quad \mathbb{U}=\mathbf{u}_{0}\left[\begin{array}{ll}
1 & \mathbf{1}^{T}
\end{array}\right]+\frac{\Delta t}{2} \mathbb{V}\left[\begin{array}{cc}
0 & \mathbf{1}^{T} \\
\mathbf{0} & \tilde{\mathbb{T}}
\end{array}\right]
$$

where the matrix $\tilde{\mathbb{T}}$ is the upper triangular matrix

$$
\tilde{\mathbb{T}}=\left[\begin{array}{cccc}
1 & 2 & \ldots & 2 \\
0 & 1 & \ddots & \vdots \\
\vdots & \ddots & \ddots & 2 \\
0 & \ldots & 0 & 1
\end{array}\right] \in \mathbb{R}^{n_{t} \times n_{t}},
$$

and $\mathbf{1}$ is a column vector of ones of dimension $n_{t}$. Similarly, the sequences $\left\{\tilde{\mathbf{a}}_{j}\right\},\left\{\tilde{\mathbf{v}}_{j}\right\}$, and $\left\{\tilde{\mathbf{u}}_{j}\right\}$ generate, respectively, the matrices $\tilde{\mathbb{A}}, \tilde{\mathbb{V}}$, and $\tilde{\mathbb{U}}$. Relation (3.11) yields

$$
\tilde{\mathbb{V}}=\tilde{\mathbf{v}}_{0}\left[\begin{array}{ll}
1 & \mathbf{1}^{T}
\end{array}\right]+\frac{\Delta t}{2} \tilde{\mathbb{A}}\left[\begin{array}{cc}
0 & \mathbf{1}^{T} \\
\mathbf{0} & \tilde{\mathbb{T}}
\end{array}\right] \quad \text { and } \quad \tilde{\mathbb{U}}=\tilde{\mathbf{u}}_{0}\left[\begin{array}{ll}
1 & \mathbf{1}^{T}
\end{array}\right]+\frac{\Delta t}{2} \tilde{\mathbb{V}}\left[\begin{array}{ll}
0 & \mathbf{1}^{T} \\
\mathbf{0} & \tilde{\mathbb{T}}
\end{array}\right] .
$$

In the previous analysis, a reduced basis is used for approximating the increments to the initial velocity and displacement. Three choices of snapshots are studied, namely

- $\mathbf{S}^{(1)}=\frac{1}{\tau}(\mathbb{U}-\tilde{\mathbb{U}})$

- $\mathbf{S}^{(2)}=\left[\frac{1}{\tau}(\mathbb{U}-\tilde{\mathbb{U}}), \mathbb{V}-\tilde{\mathbb{V}}\right]$

- $\mathbf{S}^{(3)}=\left[\frac{1}{\tau}(\mathbb{U}-\tilde{\mathbb{U}}), \mathbb{V}-\tilde{\mathbb{V}}, \tau(\mathbb{A}-\tilde{\mathbb{A}})\right]$

$\left(\lambda_{i}^{(j)}\right)_{1 \leq i \leq r^{(j)}}$ will denote the non-zero eigenvalues of the correlation matrix $\mathbf{C}^{(j)}=\left(\mathbf{S}^{(j)}\right)^{T} \mathbf{M} \mathbf{S}^{(j)}$, sorted decreasingly. To shorten the error equations, the absolute projection error in (3.13) is decomposed into the parts

$$
\begin{aligned}
e_{v}^{2} & =\sum_{j=0}^{n_{t}} \|\left(\left(\mathbf{I}-\hat{\mathbf{V}}\left(\hat{\mathbf{V}}^{T} \mathbf{M} \hat{\mathbf{V}}\right)^{-1} \hat{\mathbf{V}}^{T} \mathbf{M}\right)\left(\mathbf{v}_{j}-\tilde{\mathbf{v}}_{j}\right) \|_{\mathbf{M}}^{2}\right. \\
& =\sum_{j=0}^{n_{t}}\left\|\left(\mathbf{I}-\boldsymbol{\Pi}_{\hat{\mathbf{V}}}\right)\left(\mathbf{v}_{j}-\tilde{\mathbf{v}}_{j}\right)\right\|_{\mathbf{M}}^{2}=\left\|\mathbf{M}^{\frac{1}{2}}\left(\mathbf{I}-\boldsymbol{\Pi}_{\hat{\mathbf{V}}}\right)(\mathbb{V}-\tilde{\mathbb{V}})\right\|_{F}^{2}
\end{aligned}
$$

and

$$
\begin{aligned}
e_{u}^{2} & =\frac{1}{\tau^{2}} \sum_{j=0}^{n_{t}} \|\left(\left(\mathbf{I}-\hat{\mathbf{V}}\left(\hat{\mathbf{V}}^{T} \mathbf{M} \hat{\mathbf{V}}\right)^{-1} \hat{\mathbf{V}}^{T} \mathbf{M}\right)\left(\mathbf{u}_{j}-\tilde{\mathbf{u}}_{j}\right) \|_{\mathbf{M}}^{2}\right. \\
& =\frac{1}{\tau^{2}}\left\|\mathbf{M}^{\frac{1}{2}}\left(\mathbf{I}-\mathbf{\Pi}_{\hat{\mathbf{V}}}\right)(\mathbb{U}-\tilde{\mathbb{U}})\right\|_{F}^{2}
\end{aligned}
$$

where $\hat{\mathbf{V}}$ is defining the reduced basis. 
Considering the three different choices of snapshots, the errors (3.17) and (3.18) are bounded in terms of the corresponding eigenvalues $\lambda_{i}^{(j)}$ as follows.

- Choice of snapshots $\mathbf{S}^{(1)}$ : the proper orthogonal decomposition generates a matrix $\hat{\mathbf{V}}$ of rank $k$ such that

$$
\min _{\operatorname{rank}(\hat{\mathbf{V}})=k}\left\|\mathbf{M}^{\frac{1}{2}}\left(\mathbf{I}-\mathbf{\Pi}_{\hat{\mathbf{V}}}\right) \mathbf{S}^{(1)}\right\|_{F}=\sqrt{\sum_{i=k+1}^{r^{(1)}} \lambda_{i}^{(1)}}
$$

The projection error (3.18) verifies

$$
\begin{aligned}
e_{u}^{2}=\frac{1}{\tau^{2}}\left\|\mathbf{M}^{\frac{1}{2}}\left(\mathbf{I}-\mathbf{\Pi}_{\hat{\mathbf{V}}}\right)(\mathbb{U}-\tilde{\mathbb{U}})\right\|_{F}^{2}=\left\|\mathbf{M}^{\frac{1}{2}}\left(\mathbf{I}-\mathbf{\Pi}_{\hat{\mathbf{V}}}\right) \mathbf{S}^{(1)}\right\|_{F}^{2}=\sum_{i=k+1}^{r^{(1)}} \lambda_{i}^{(1)} & \\
& =\left(\frac{\sum_{i=k+1}^{r^{(1)}} \lambda_{i}^{(1)}}{\sum_{i=1}^{r^{(1)}} \lambda_{i}^{(1)}}\right) \frac{1}{\tau^{2}}\left\|\mathbf{M}^{\frac{1}{2}}(\mathbb{U}-\tilde{\mathbb{U}})\right\|_{F}^{2}
\end{aligned}
$$

The property,

$$
\Pi_{\hat{\mathbf{V}}}^{T} \mathbf{M} \Pi_{\hat{\mathbf{V}}}=\mathbf{M} \Pi_{\hat{\mathbf{V}}}=\Pi_{\hat{\mathbf{V}}}^{T} \mathbf{M}
$$

gives

$$
e_{v}^{2}=\left\|\mathbf{M}^{\frac{1}{2}}\left(\mathbf{I}-\mathbf{\Pi}_{\hat{\mathbf{V}}}\right)(\mathbb{V}-\tilde{\mathbb{V}})\right\|_{F}^{2} \leq\left\|\mathbf{M}^{\frac{1}{2}}(\mathbb{V}-\tilde{\mathbb{V}})\right\|_{F}^{2},
$$

indicating that the projection error remains bounded when $\Delta t$ goes to 0 ,

$$
\lim _{\Delta t \rightarrow 0} \Delta t\left\|\mathbf{M}^{\frac{1}{2}}\left(\mathbf{I}-\mathbf{\Pi}_{\hat{\mathbf{V}}}\right)(\mathbb{V}-\tilde{\mathbb{V}})\right\|_{F}^{2} \leq \lim _{\Delta t \rightarrow 0} \Delta t\left\|\mathbf{M}^{\frac{1}{2}}(\mathbb{V}-\tilde{\mathbb{V}})\right\|_{F}^{2}=\int_{0}^{T}\left\|\frac{\mathrm{d} \mathbf{u}}{\mathrm{d} t}(t)-\frac{\mathrm{d} \tilde{\mathbf{u}}}{\mathrm{d} t}(t)\right\|_{\mathbf{M}}^{2} \mathrm{~d} t .
$$

In addition, relations (3.14) and (3.16) yield

$$
\begin{aligned}
& \mathbb{U}-\tilde{\mathbb{U}}=\left(\mathbf{u}_{0}-\tilde{\mathbf{u}}_{0}\right)\left[\begin{array}{ll}
1 & \mathbf{1}^{T}
\end{array}\right]+\frac{\Delta t}{2}(\mathbb{V}-\tilde{\mathbb{V}})\left[\begin{array}{ll}
0 & 1^{T} \\
0 & \tilde{\mathbb{T}}
\end{array}\right], \\
& \mathbb{U}-\tilde{\mathbb{U}}=\left(\mathbf{u}_{0}-\tilde{\mathbf{u}}_{0}\right)\left[\begin{array}{ll}
1 & \mathbf{1}^{T}
\end{array}\right]+\frac{\Delta t}{2}(\mathbb{V}-\tilde{\mathbb{V}})\left[\begin{array}{cc}
1 & 0 \\
\mathbf{0} & \tilde{\mathbb{T}}
\end{array}\right]+\frac{\Delta t}{2}\left(\mathbf{v}_{0}-\tilde{\mathbf{v}}_{0}\right)\left[\begin{array}{ll}
-1 & \left.\mathbf{1}^{T}\right]
\end{array}\right.
\end{aligned}
$$

and

$$
\mathbb{V}-\tilde{\mathbb{V}}=\frac{2}{\Delta t}(\mathbb{U}-\tilde{\mathbb{U}})\left[\begin{array}{cc}
1 & \mathbf{0} \\
\mathbf{0} & \tilde{\mathbb{T}}^{-1}
\end{array}\right]-\frac{2}{\Delta t}\left(\mathbf{u}_{0}-\tilde{\mathbf{u}}_{0}\right)\left[\begin{array}{ll}
1 & \mathbf{1}^{T} \tilde{\mathbb{T}}^{-1}
\end{array}\right]-\left(\mathbf{v}_{0}-\tilde{\mathbf{v}}_{0}\right)\left[\begin{array}{ll}
-1 & \mathbf{1}^{T} \tilde{\mathbb{T}}^{-1}
\end{array}\right] .
$$

By combining the previous equality and (3.19), the projection error $e_{v}$ then satisfies

$$
\begin{aligned}
e_{v}^{2}=\left\|\mathbf{M}^{\frac{1}{2}}\left(\mathbf{I}-\mathbf{\Pi}_{\hat{\mathbf{V}}}\right)(\mathbb{V}-\tilde{\mathbb{V}})\right\|_{F}^{2} \leq C \kappa(\tilde{\mathbb{T}})^{2}\left(\frac{\sum_{i=k+1}^{r^{(1)}} \lambda_{i}^{(1)}}{\sum_{i=1}^{r^{(1)}} \lambda_{i}^{(1)}}\right)\left\|\mathbf{M}^{\frac{1}{2}}(\mathbb{V}-\tilde{\mathbb{V}})\right\|_{F}^{2} \\
+C\left(\frac{\kappa(\tilde{\mathbb{T}})}{\Delta t}\right)^{2}\left\|\mathbf{M}^{\frac{1}{2}}\left(\mathbf{I}-\mathbf{\Pi}_{\hat{\mathbf{V}}}\right)\left(\mathbf{u}_{0}-\tilde{\mathbf{u}}_{0}\right)\right\|_{F}^{2}+C \kappa(\tilde{\mathbb{T}})^{2}\left\|\mathbf{M}^{\frac{1}{2}}\left(\mathbf{I}-\mathbf{\Pi}_{\hat{\mathbf{V}}}\right)\left(\mathbf{v}_{0}-\tilde{\mathbf{v}}_{0}\right)\right\|_{F}^{2},
\end{aligned}
$$

where the constant $C$ does not depend on $\Delta t$ and $\kappa(\tilde{\mathbb{T}})$ denotes the condition number of the matrix $\tilde{\mathbb{T}}$ for the Frobenius norm. The projection error $e_{v}$ is therefore bounded by the minimum between (3.20) and (3.23). 
- Choice of snapshots $\mathbf{S}^{(2)}$ : a proper orthogonal decomposition creates a matrix $\hat{\mathbf{V}}$ of rank $k$ such that

$$
\min _{\operatorname{rank}(\hat{\mathbf{V}})=k}\left\|\mathbf{M}^{\frac{1}{2}}\left(\mathbf{I}-\mathbf{\Pi}_{\hat{\mathbf{V}}}\right) \mathbf{S}^{(2)}\right\|_{F}=\sqrt{\sum_{i=k+1}^{r^{(2)}} \lambda_{i}^{(2)}} .
$$

By definition of $\mathbf{S}^{(2)}$, the projection errors satisfy

$$
\begin{aligned}
e_{u}^{2}+e_{v}^{2}=\frac{1}{\tau^{2}}\left\|\mathbf{M}^{\frac{1}{2}}\left(\mathbf{I}-\mathbf{\Pi}_{\hat{\mathbf{V}}}\right)(\mathbb{U}-\tilde{\mathbb{U}})\right\|_{F}^{2} & +\left\|\mathbf{M}^{\frac{1}{2}}\left(\mathbf{I}-\mathbf{\Pi}_{\hat{\mathbf{V}}}\right)(\mathbb{V}-\tilde{\mathbb{V}})\right\|_{F}^{2}=\sum_{i=k+1}^{r^{(2)}} \lambda_{i}^{(2)} \\
& =\left(\frac{\sum_{i=k+1}^{r^{(2)}} \lambda_{i}^{(2)}}{\sum_{i=1}^{r^{(2)}} \lambda_{i}^{(2)}}\right)\left(\frac{1}{\tau^{2}}\left\|\mathbf{M}^{\frac{1}{2}}(\mathbb{U}-\tilde{\mathbb{U}})\right\|_{F}^{2}+\left\|\mathbf{M}^{\frac{1}{2}}(\mathbb{V}-\tilde{\mathbb{V}})\right\|_{F}^{2}\right) .
\end{aligned}
$$

- Choice of snapshots $\mathbf{S}^{(3)}$ : the proper orthogonal decomposition generates a matrix $\hat{\mathbf{V}}$ of rank $k$ such that

$$
\min _{\operatorname{rank}(\hat{\mathbf{V}})=k}\left\|\mathbf{M}^{\frac{1}{2}}\left(\mathbf{I}-\mathbf{\Pi}_{\hat{\mathbf{V}}}\right) \mathbf{S}^{(3)}\right\|_{F}=\sqrt{\sum_{i=k+1}^{r^{(3)}} \lambda_{i}^{(3)}} .
$$

For this case, the projection errors satisfy

$$
\begin{aligned}
e_{u}^{2}+e_{v}^{2}=\frac{1}{\tau^{2}}\left\|\mathbf{M}^{\frac{1}{2}}\left(\mathbf{I}-\boldsymbol{\Pi}_{\hat{\mathbf{V}}}\right)(\mathbb{U}-\tilde{\mathbb{U}})\right\|_{F}^{2}+\left\|\mathbf{M}^{\frac{1}{2}}\left(\mathbf{I}-\mathbf{\Pi}_{\hat{\mathbf{V}}}\right)(\mathbb{V}-\tilde{\mathbb{V}})\right\|_{F}^{2} \\
\quad \leq\left(\frac{\sum_{i=k+1}^{r^{(3)}} \lambda_{i}^{(3)}}{\sum_{i=1}^{r^{(3)}} \lambda_{i}^{(3)}}\right)\left(\frac{1}{\tau^{2}}\left\|\mathbf{M}^{\frac{1}{2}}(\mathbb{U}-\tilde{\mathbb{U}})\right\|_{F}^{2}+\left\|\mathbf{M}^{\frac{1}{2}}(\mathbb{V}-\tilde{\mathbb{V}})\right\|_{F}^{2}+\tau^{2}\left\|\mathbf{M}^{\frac{1}{2}}(\mathbb{A}-\tilde{\mathbb{A}})\right\|_{F}^{2}\right)
\end{aligned}
$$

because $\left[\frac{1}{\tau}(\mathbb{U}-\tilde{\mathbb{U}}), \mathbb{V}-\tilde{\mathbb{V}}\right]$ is a subset of the columns of $\mathbf{S}^{(3)}$.

All these estimates highlight that the norms for the projection errors, when using a set of snapshots composed either of (1) displacement snapshots, (2) velocities and of displacements snapshots and (3) a set of acceleration, velocity and displacement snapshots, are bounded in terms of the corresponding neglected singular values.

In the case of snapshots based on displacements only, the bounds in Theorem 3.3 and the bounds on the projection error norms remain bounded when $\Delta t$ goes to 0 . This result is an improvement over the one described by Herkt et al. in [14] A comparison of the estimates for the second and third choices of snapshots also highlights the fact that adding acceleration snapshots, as suggested by Herkt and coworkers in [14], does not add information that leads to better projection error estimates for velocity and displacement.

\section{NumericAl EXPERIMENTS}

In this section, numerical experiments are conducted for three different application systems. The objective is to illustrate the theoretical results obtained for the semi-discrete wave equation. The accuracy of the reduced models constructed using (1) the three snapshots collections presented above and (2) eigendecomposition are studied by computing the total relative error

$$
e_{\mathrm{ROM}}(k)=\sqrt{\frac{\sum_{j=0}^{n_{t}}\left\|\mathbf{v}_{j}-\tilde{\mathbf{v}}_{j}-\hat{\mathbf{V}} \hat{\mathbf{v}}_{j}\right\|_{\mathbf{M}}^{2}+\frac{1}{\tau^{2}}\left\|\mathbf{u}_{j}-\tilde{\mathbf{u}}_{j}-\hat{\mathbf{V}} \hat{\mathbf{u}}_{j}\right\|_{\mathbf{M}}^{2}}{\sum_{j=0}^{n_{t}}\left\|\mathbf{v}_{j}\right\|_{\mathbf{M}}^{2}+\frac{1}{\tau^{2}}\left\|\mathbf{u}_{j}\right\|_{\mathbf{M}}^{2}}},
$$




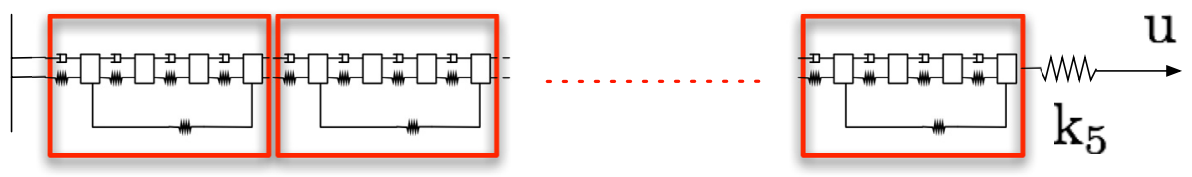

Figure 1. Chain of 20 mass-spring-damper blocks.

with $\tilde{\mathbf{v}}_{j}=\mathbf{v}_{0}$ and $\tilde{\mathbf{u}}_{j}=\mathbf{u}_{0}+j \Delta t \mathbf{v}_{0}$, the total projection error

$$
\begin{aligned}
& e_{\mathrm{ROM}, p}(k)= \\
& \sqrt{\frac{\sum_{j=0}^{n_{t}}\left\|\mathbf{v}_{j}-\tilde{\mathbf{v}}_{j}-\hat{\mathbf{V}}\left(\hat{\mathbf{V}}^{T} \mathbf{M} \hat{\mathbf{V}}\right)^{-1} \hat{\mathbf{V}}^{T} \mathbf{M}\left(\mathbf{v}_{j}-\tilde{\mathbf{v}}_{j}\right)\right\|_{\mathbf{M}}^{2}+\frac{1}{\tau^{2}}\left\|\mathbf{u}_{j}-\tilde{\mathbf{u}}_{j}-\hat{\mathbf{V}}\left(\hat{\mathbf{V}}^{T} \mathbf{M} \hat{\mathbf{V}}\right)^{-1} \hat{\mathbf{V}}^{T} \mathbf{M}\left(\mathbf{u}_{j}-\tilde{\mathbf{u}}_{j}\right)\right\|_{\mathbf{M}}^{2}}{\sum_{j=0}^{n_{t}}\left\|\mathbf{v}_{j}\right\|_{\mathbf{M}}^{2}+\frac{1}{\tau^{2}}\left\|\mathbf{u}_{j}\right\|_{\mathbf{M}}^{2}}},
\end{aligned}
$$

their ratio

$$
\frac{e_{\mathrm{ROM}}(k)}{e_{\mathrm{ROM}, p}(k)}
$$

the two components of the relative error:

$$
e_{\mathrm{ROM}, u}(k)=\sqrt{\frac{\frac{1}{\tau^{2}} \sum_{j=0}^{n_{t}}\left\|\mathbf{u}_{j}-\tilde{\mathbf{u}}_{j}-\hat{\mathbf{V}} \hat{\mathbf{u}}_{j}\right\|_{\mathbf{M}}^{2}}{\sum_{j=0}^{n_{t}}\left\|\mathbf{v}_{j}\right\|_{\mathbf{M}}^{2}+\frac{1}{\tau^{2}}\left\|\mathbf{u}_{j}\right\|_{\mathbf{M}}^{2}}}
$$

and

$$
e_{\mathrm{ROM}, v}(k)=\sqrt{\frac{\sum_{j=0}^{n_{t}}\left\|\mathbf{v}_{j}-\tilde{\mathbf{v}}_{j}-\hat{\mathbf{V}} \hat{\mathbf{v}}_{j}\right\|_{\mathbf{M}}^{2}}{\sum_{j=0}^{n_{t}}\left\|\mathbf{v}_{j}\right\|_{\mathbf{M}}^{2}+\frac{1}{\tau^{2}}\left\|\mathbf{u}_{j}\right\|_{\mathbf{M}}^{2}} .}
$$

The projection error can serve as an error predictor for the reduced-order model, provided the error ratio does not increase drastically when the size $k$ of the model increases. In that case, the truncation can be used to determine a priori the increase in dimension of the reduced-order model (ROM) to achieve a given error reduction.

\subsection{Discrete mass-spring-damper system}

The first system of interest is a chain of 20 discrete systems constituted of masses, dampers and springs. This chain is depicted in Figure 1. An impulse force is applied to the mass at the extremity of the chain. The configuration of each block is represented in Figure 2. Finally, the physical characteristics of each mass, spring and damper are reported in Table 1.

The system has 80 displacement degrees of freedom and 80 velocity degrees of freedom. Time integration is performed using the Newmark scheme with equidistant time-step $\Delta t=0.1 \mathrm{~s}$. Here $\tau=1 \mathrm{~s} . n_{t}=50$ snapshots are generated by applying an impulse input elongation $u$ to the right extremity of the spring of stiffness $k_{5}$ 


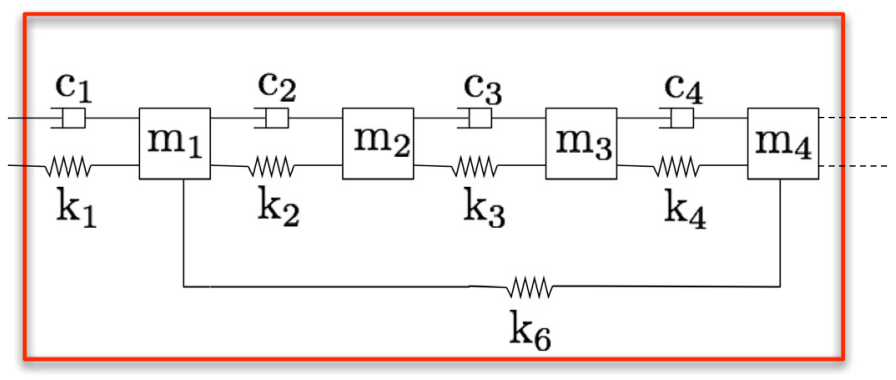

FiguRE 2. Mass-spring-damper block configuration.

TABLE 1. Physical properties of the mass-damper-spring elements.

\begin{tabular}{|c|c|c|c|c|c|}
\hline \multicolumn{2}{|c|}{ Masses } & \multicolumn{2}{|c|}{ Dampers } & \multicolumn{2}{|c|}{ Springs } \\
\hline & $(\mathrm{kg})$ & & $\overline{\mathrm{Ns} / \mathrm{m})}$ & & $(\mathrm{N} / \mathrm{m})$ \\
\hline$m_{1}$ & 125 & $c_{1}$ & 5 & $k_{1}$ & 12 \\
\hline$m_{2}$ & 25 & $c_{2}$ & 1.6 & $k_{2}$ & 1 \\
\hline$m_{3}$ & 5 & $c_{3}$ & 0.4 & $k_{3}$ & 3 \\
\hline$m_{4}$ & 1 & $c_{4}$ & 0.1 & $k_{4}$ & 9 \\
\hline & & & & $k_{5}$ & 27 \\
\hline & & & & $k_{6}$ & 11 \\
\hline
\end{tabular}

attached to the extremal block. Nonzero initial conditions (displacement $\mathbf{u}_{0}$ and velocity $\mathbf{v}_{0}$ ) are considered: the initial displacement vector is $\mathbf{1}$ and the initial velocity profile is linear, the left-most mass having zero initial velocity and the right-most mass unitary initial velocity.

A comparison of the errors, projection errors and their ratios associated with each snapshot collection is reported in Figure 3. Similar results are also reported for ROMs based on modal truncation (eigendecomposition). The error associated with the reduced-order models based on POD decreases sharply in all three cases, reaching $10^{-8}$ relative error for ROMs of dimension $k=12$. On the other hand, ROMs based on eigendecomposition lead to much less accurate predictions, with errors of the order of $10 \%$. Adding eigenmodes does not improve the error for ROMs of dimension smaller than 12. This illustrates the fact that, as opposed to ROMs based on POD, ROMs based on modal truncation are not trained for a specific scenario and require a large basis size to achieve high accuracy. In the present case, all snapshot choices lead to similar accuracy for a given size $k$. Furthermore, the projection error appears to be a good a priori indicator of the error. The error decomposition in the case of snapshots based on displacement and velocity is also reported in Figure 3.

The error ratio for ROMs based on the eigendecomposition is equal to one. This observation is in agreement with the first remark in Section 2.1 and Theorem 3.1. It shows that the error between the ROM and the underlying high-fidelity model is equal to the projection error. However, this projection error can be large, as illustrated in the present case.

\subsection{Butterfly gyro system}

The second set of numerical experiments is carried on a butterfly gyro MEMS originally developed and presented by Lienemann and coworkers in [26]. The full-order linear operators associated with this model are available as part of the Oberwolfach model depository [29]. A Rayleigh damping of the form $\mathbf{D}=10^{-6} \times \mathbf{K}$ is chosen. The physical model is discretized by the finite elements method, resulting in 17,361 displacement degrees of freedom. The system input applies simultaneously eight distinct voltages to the MEMS. Four detections electrodes recording local structural displacements are also defined. Time integration is performed by 

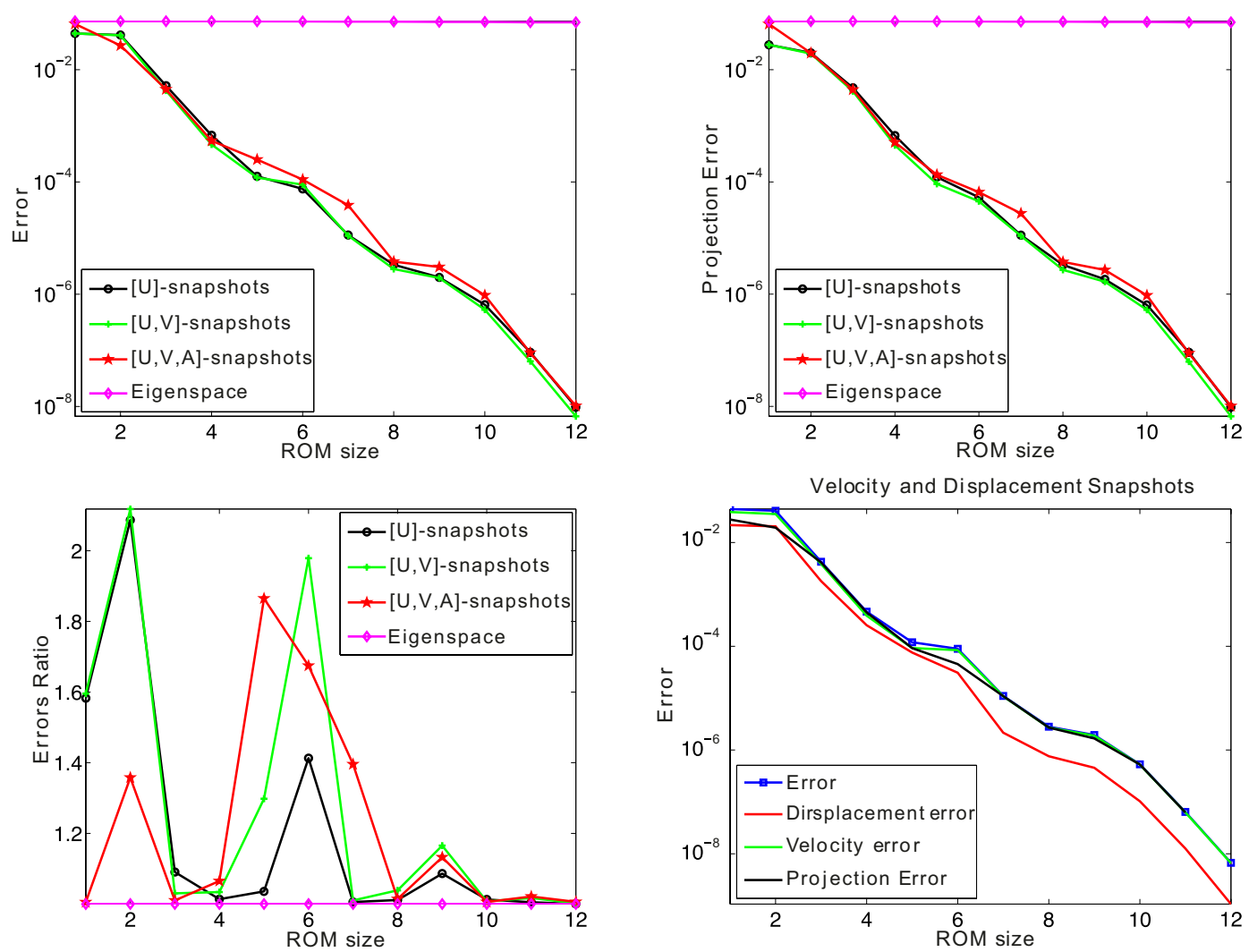

FiguRE 3. Comparison for the mass-spring-damper system of the error (top left), projection error (top right) and errors ratio (bottom left) obtained with each model reduction approach, as well as the error decomposition in the case of displacement and velocity snapshots (bottom right).

the Newmark scheme with a constant time step $\Delta t=7.5 \times 10^{-6} \mathrm{~s}$. A step input vector (DC voltages) is here applied with zero initial conditions, resulting in the collection of $n_{t}=200$ snapshots. A choice $\tau=1 \mathrm{~s}$ in made.

The displacements of the center of the first detection electrode [29] obtained using (1) the high-dimensional finite element model of dimension 17,361 and (2) the reduced-order models of dimension 10 generated using each method are first compared in Figure 4. The reader can observe that each ROM leads to very accurate responses when compared to the underlying high-fidelity model except for the displacement of the electrode in the $x$-direction obtained with the ROM that was generated using displacement, velocity and acceleration snapshots. The best predictions are here obtained using the ROM generated from displacement snapshots.

A comparison of the errors, projection errors and their ratios is provided in Figure 5. One can observe that, in most cases, the most accurate ROMs result from a choice of displacement and velocity snapshots. POD based on velocity, displacement and acceleration snapshots is the snapshot-based method resulting in the least accurate predictions. Interestingly, the accuracy of ROMs based on eigenvalue decomposition improves sharply only when certain modes are added to the basis, such as mode 5 and 15. The associated ROMs are however less accurate in general than their POD-based counterparts.

The projection error provides accurate a priori error estimates, except for ROMs of small dimension based on displacement and the three sets of snapshots. The error decomposition shows that the total error is dominated by the velocity error term. 

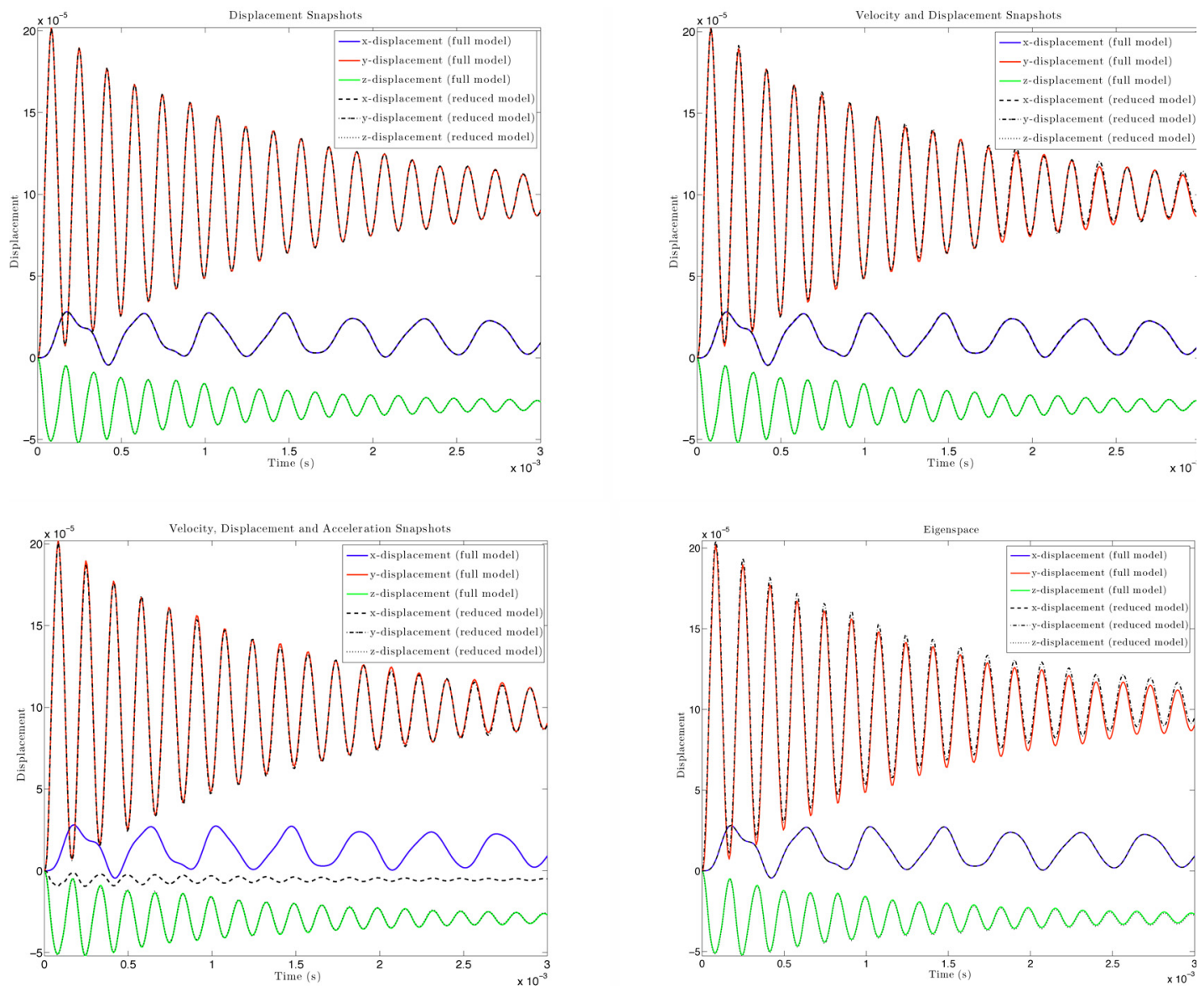

Figure 4. Time responses of the displacement at a sensor location: comparison between the full model response and the responses of each reduced-order model of dimension $k=10$ obtained using the following approaches: POD using displacement snapshots (top left), displacement and velocity snapshots (top right), displacement, velocity and acceleration snapshots (bottom left) and eigendecomposition (bottom right).

\subsection{Synthetic system}

A third set of numerical experiments is carried on to study the effect of space discretization onto the error estimation. For that purpose, a synthetic example, initially studied by Herkt and coworkers in [14] is considered. The system stems from the discretization of the continuous wave equation

$$
\begin{aligned}
\frac{\partial^{2} y}{\partial t^{2}}(x, t)-\frac{\partial^{2} y}{\partial x^{2}}(x, t) & =0,(x, t) \in[0,1] \times[0, T], \\
y(x, t) & =0, x \in\{0,1\} .
\end{aligned}
$$

Appropriate initial conditions are chosen such that the solution to the system is

$$
y(x, t)=\sum_{i=1}^{21} \sin (i \pi x) \cos (i \pi t) .
$$



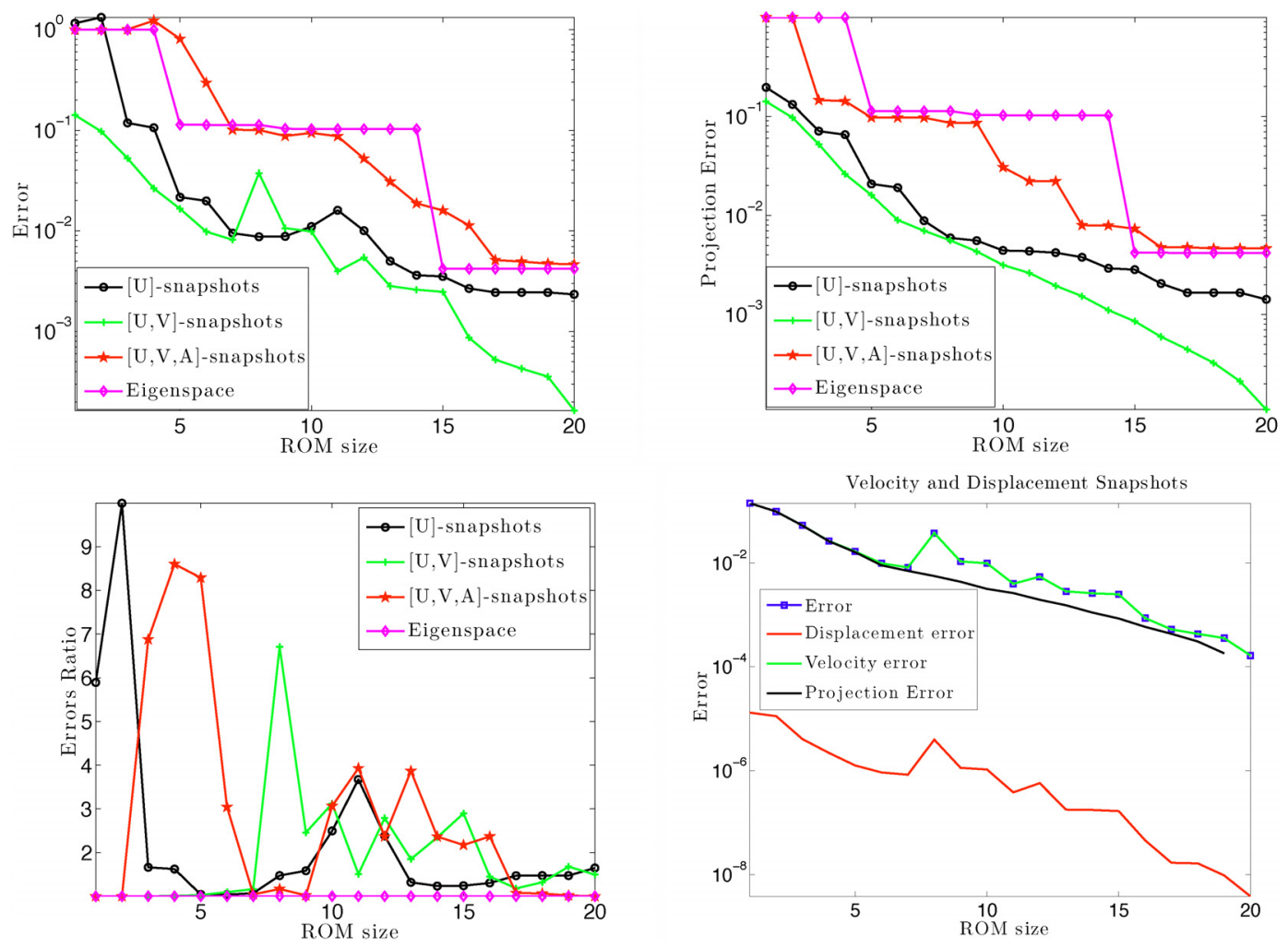

FiguRE 5. Comparison for the gyro system of the error (top left), projection error (top right) and errors ratio (bottom left) obtained with each model reduction approach, as well as the error decomposition in the case of displacement and velocity snapshots (bottom right).

The system is discretized in space by the finite element method with piecewise linear elements. In a first set of experiments, a discretization with $N=128$ elements is used. The system is then discretized in space by the Newmark scheme using an equidistant time step $\Delta t=0.0078 \mathrm{~s}$ and $\tau=1 \mathrm{~s}$. Two final times are considered: (a) $T=2 \mathrm{~s}$, which corresponds to one period for the solution, (b) $T=0.4 \mathrm{~s}$.

When $T=2 \mathrm{~s}, 256$ displacement, velocity and acceleration snapshots are collected, respectively. The four model reduction approaches considered are applied and the corresponding errors, projection errors and ratios reported in Figure 6. The most accurate ROMs are the ones containing the velocity snapshots, as ROMs based on displacement snapshots only lead to poor predictions, similar to the ones based on modal truncation. By construction, all ROMs of dimension $k=21$ lead to perfect accuracy. The projection error a priori estimates show perfect agreement with the error, except in the case of displacement snapshots, for which the agreement is fair. This means that the error between the ROMs and the high-dimensional model in the subspace spanned by the reduced-order bases is negligible when compared to the projection error.

Next, the same numerical experiments are performed when $T=0.4 \mathrm{~s}$, leading to the collection of 51 snapshots. In that case, all POD-based ROMs lead to a similar level of accuracy for a given choice of ROM dimension $k$. On the other hand, modal truncation-based ROMs are much less accurate for $k<21$. The projection error is in good agreement with the error, except in the case of large ROMs, for which the errors are close to machine precision. Once again, the total error is dominated by its velocity component.

Figure 8 illustrates the behavior of the ratio between the total relative error (4.1) and the total projection error (4.2) when $T=2 \mathrm{~s}$ as the mesh size and the time steps are decreased. The dimension of the reduced-order 

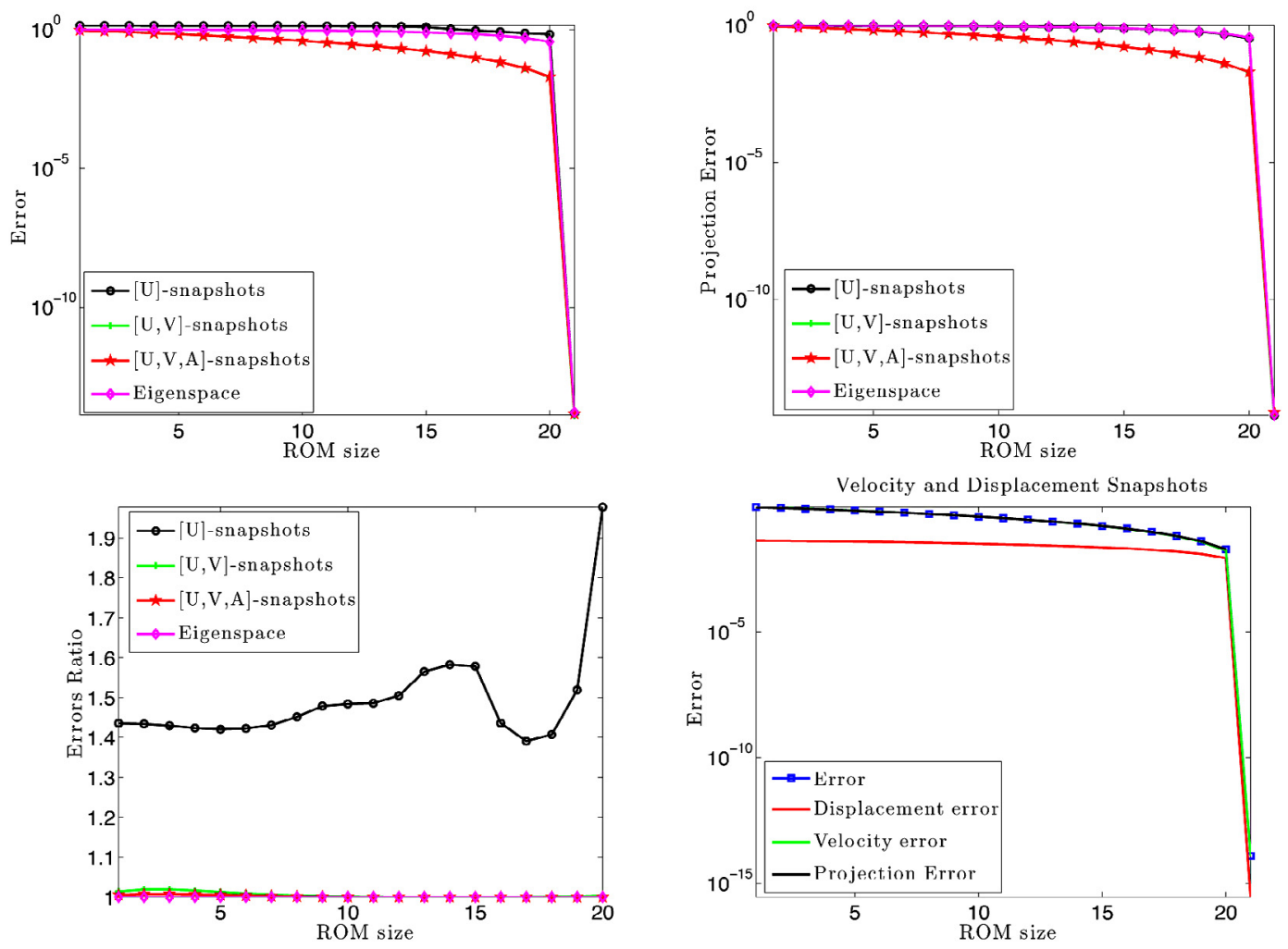

Figure 6. Comparison for the synthetic system with $T=2$ of the error (top left), projection error (top right) and errors ratio (bottom left) obtained with each model reduction approach, as well as the error decomposition in the case of displacement and velocity snapshots (bottom right).

models is set at 18 and the relative projection errors (4.2) are between $6 \%$ and $60 \%$. For a fixed mesh size, the ratios appear to converge to a fixed value as the time steps are decreased. Only the reduced order models built with displacement snapshots yield a ratio different from 1. These asymptotic plateaus do not appear to depend on the mesh size. However, for the reduced order models built with displacement snapshots, the critical time step below which the ratio is constant depends on the mesh size. A finer mesh size requires a smaller critical time step, even though the time integration scheme is implicit. This experiment illustrates that models built on displacements snapshots do not behave, with respect to the mesh size, as robustly as the models built with the other sets of snapshots.

\section{Conclusions}

Reduced-order models are an efficient tool for simulating dynamical systems at a reduced cost. Theoretical results on the state approximation error have so far been focused on first-order dynamical systems. The extension of these analyses to the second-order semi-discrete wave equation requires special attention to preserve the original structure and to retain the physical interpretation of the reduced variables.

In this work, error bounds for the full state variables of the semi-discrete wave equation have been derived in the continuous setting (when the whole trajectory is known) and in the discrete setting when the Newmark average-acceleration scheme is used. When the approximating subspace is constructed using the proper orthogonal decomposition, the error estimates are proportional to the sums of the neglected singular values. Numerical 

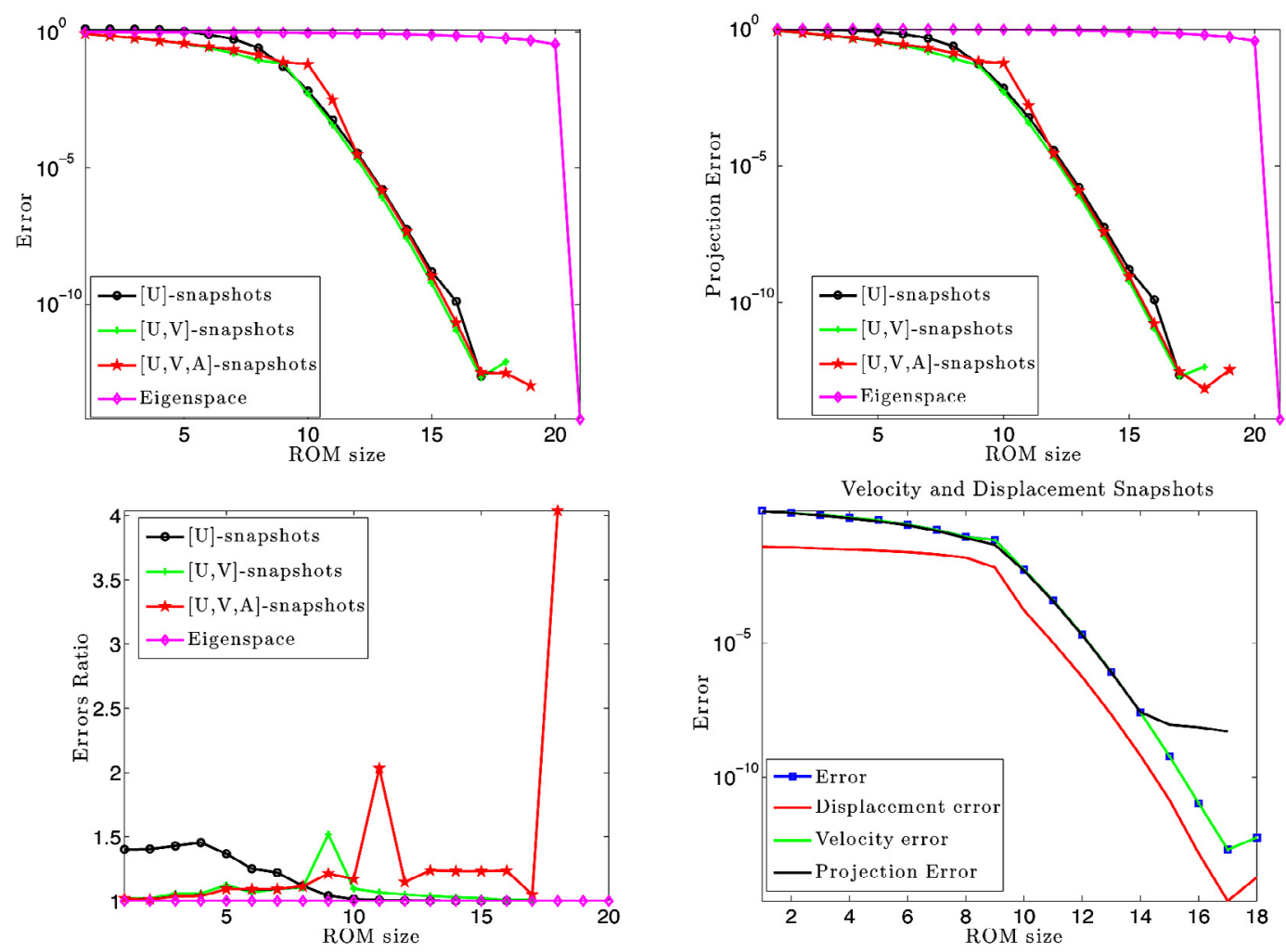

Figure 7. Comparison for the synthetic system with $T=0.4$ of the error (top left), projection error (top right) and errors ratio (bottom left) obtained with each model reduction approach, as well as the error decomposition in the case of displacement and velocity snapshots (bottom right).

experiments illustrate the theoretical results when the choice of snapshots is set heuristically. Some procedures for automatic selection of these snapshots exist in the literature $[9,24,25]$.

\section{Appendix A. Proofs}

\section{A.1. Proof of Theorem 2.3}

The bound in this theorem relies on a Gronwall-like inequality. Consider the sequence $\left(\phi_{n}\right)_{0 \leq n \leq n_{t}}$ such that

$$
0 \leq \phi_{n} \leq \beta \phi_{n-1}+\alpha \Delta t p_{n}+\alpha \Delta t p_{n-1}, \quad \text { for } n \geq 1,
$$

where $\beta \geq 1$ and $\alpha$ is a non-negative number. The sequence $\left(p_{n}\right)_{0 \leq n \leq n_{t}}$ is made of non-negative numbers. For $1 \leq n \leq n_{t}, \phi_{n}$ satisfies

$$
\phi_{n} \leq \beta^{n} \phi_{0}+\alpha \Delta t p_{j}+\alpha \Delta t(1+\beta) \sum_{i=0}^{n-1} \beta^{n-1-i} p_{i}, \quad \text { for } n \geq 1
$$




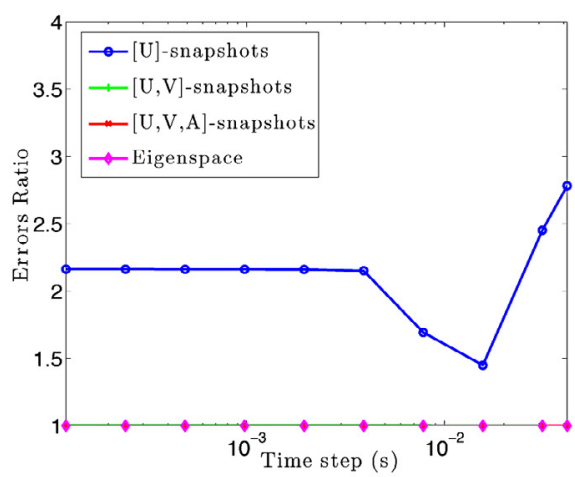

(a) $h=\frac{1}{32}$

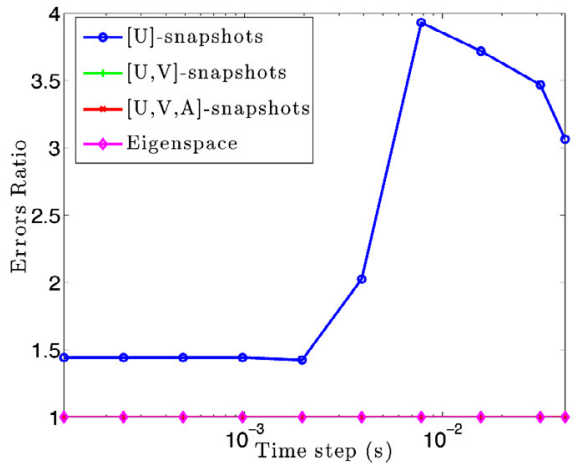

(c) $h=\frac{1}{512}$

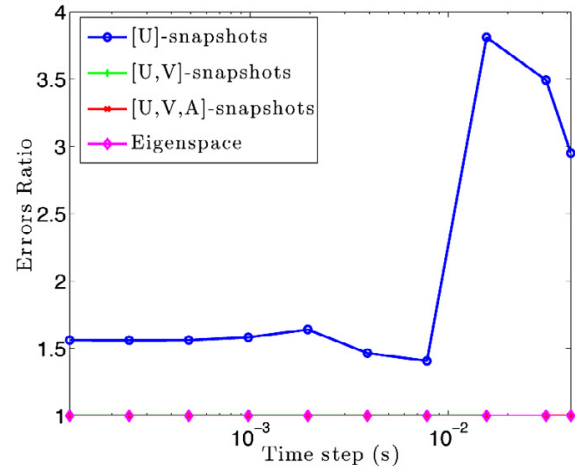

(b) $h=\frac{1}{128}$

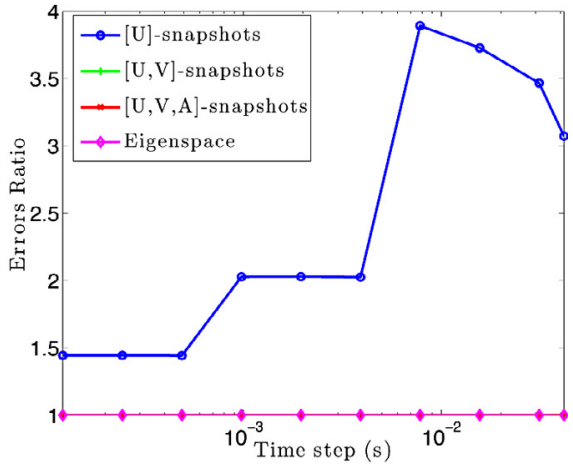

(d) $h=\frac{1}{2048}$

Figure 8. Comparison for the synthetic system of the errors ratio obtained with each model reduction approach when the space and time discretizations are varied.

The proof of (A.1) is made by mathematical induction. The estimate is valid for $n=1$ (because $1 \leq 1+\beta$ ). Assume it is is satisfied up to $n-1$, then $\phi_{n}$ satisfies

$$
\phi_{n} \leq \beta \phi_{n-1}+\alpha \Delta t p_{n}+\alpha \Delta t p_{n-1} \leq \beta\left(\beta^{n-1} \phi_{0}+\alpha \Delta t p_{n-1}+\alpha \Delta t(1+\beta) \sum_{i=0}^{n-2} \beta^{n-2-i} p_{i}\right)+\alpha \Delta t p_{n}+\alpha \Delta t p_{n-1} .
$$

Re-organizing the terms yields

$$
\phi_{n} \leq \beta^{n} \phi_{0}+\alpha \Delta t p_{n}+\alpha \Delta t(1+\beta) p_{n-1}+\alpha \Delta t(1+\beta) \sum_{i=0}^{n-2} \beta^{n-1-i} p_{i},
$$

which concludes the induction. Equation (A.1) can be rewritten as follows

$$
\phi_{n} \leq \beta^{n} \phi_{0}+\alpha \Delta t\left(1+\frac{1}{\beta}\right) \sum_{i=0}^{n} \beta^{n-i} p_{i} .
$$

Squaring the inequality and using the Cauchy-Schwarz inequality yield

$$
\phi_{n}^{2} \leq 2 \beta^{2 n} \phi_{0}^{2}+2 \alpha^{2}(\Delta t)^{2}\left(1+\frac{1}{\beta}\right)^{2}\left(\sum_{i=0}^{n_{t}} \beta^{2 i}\right)\left(\sum_{i=0}^{n_{t}} p_{i}^{2}\right) \quad \text { for } 0 \leq n \leq n_{t}
$$


and

$$
\Delta t \sum_{j=0}^{n_{t}} \phi_{j}^{2} \leq 2 \phi_{0}^{2}\left(\Delta t \sum_{i=0}^{n_{t}} \beta^{2 i}\right)+2 \alpha^{2}(N \Delta t)\left(\Delta t \sum_{i=0}^{n_{t}} \beta^{2 i}\right)\left(\Delta t \sum_{i=0}^{n_{t}} p_{i}^{2}\right) .
$$

Recall that the part $\theta_{j}$ satisfies

$$
\frac{\theta_{j}-\theta_{j-1}}{\Delta t}=\mathbf{V}\left(\mathbf{V}^{T} \mathbf{V}\right)^{-1} \mathbf{V}^{T} \mathbf{A}\left(\frac{\rho_{j}+\rho_{j-1}}{2}+\frac{\theta_{j}+\theta_{j-1}}{2}\right)
$$

or

$$
\begin{aligned}
\theta_{j}=\left(\mathbf{I}+\Delta t\left(\mathbf{I}-\frac{\Delta t}{2} \mathbf{V}\left(\mathbf{V}^{T} \mathbf{V}\right)^{-1}\right.\right. & \left.\left.\mathbf{V}^{T} \mathbf{A}\right)^{-1} \mathbf{V}\left(\mathbf{V}^{T} \mathbf{V}\right)^{-1} \mathbf{V}^{T} \mathbf{A}\right) \theta_{j-1} \\
& +\frac{\Delta t}{2}\left(\mathbf{I}-\frac{\Delta t}{2} \mathbf{V}\left(\mathbf{V}^{T} \mathbf{V}\right)^{-1} \mathbf{V}^{T} \mathbf{A}\right)^{-1} \mathbf{V}\left(\mathbf{V}^{T} \mathbf{V}\right)^{-1} \mathbf{V}^{T} \mathbf{A}\left(\rho_{j}+\rho_{j-1}\right)
\end{aligned}
$$

The theorem is proved by selecting $\phi_{0}=0, \phi_{n}=\left\|\theta_{n}\right\|_{2}$,

$$
\begin{aligned}
& \beta=\left\|\mathbf{I}+\Delta t\left(\mathbf{I}-\frac{\Delta t}{2} \mathbf{V}\left(\mathbf{V}^{T} \mathbf{V}\right)^{-1} \mathbf{V}^{T} \mathbf{A}\right)^{-1} \mathbf{V}\left(\mathbf{V}^{T} \mathbf{V}\right)^{-1} \mathbf{V}^{T} \mathbf{A}\right\|_{2}, \\
& \alpha=\frac{1}{2}\left\|\left(\mathbf{I}-\frac{\Delta t}{2} \mathbf{V}\left(\mathbf{V}^{T} \mathbf{V}\right)^{-1} \mathbf{V}^{T} \mathbf{A}\right)^{-1} \mathbf{V}\left(\mathbf{V}^{T} \mathbf{V}\right)^{-1} \mathbf{V}^{T} \mathbf{A} \mathbf{Z}\left(\mathbf{Z}^{T} \mathbf{Z}\right)^{-1} \mathbf{Z}^{T}\right\|_{2}
\end{aligned}
$$

and $p_{n}=\left\|\rho_{n}\right\|_{2}$. Note that the definition of $\beta$ yields

$$
\beta \leq 1+\gamma \Delta t \leq \mathrm{e}^{\gamma \Delta t}(\text { with } \gamma>0) \text { and } \sum_{j=0}^{n_{t}} \beta^{2 j} \leq \sum_{j=0}^{n_{t}} \mathrm{e}^{2 \gamma j \Delta t} .
$$

\section{A.2. Proofs for Section 2.2}

Results in this section are reformulations of results in Theorems 2.1 and 2.3. The matrix $\mathcal{M}$ is symmetric positive definite and its symmetric square root $\mathcal{M}^{\frac{1}{2}}$ is obtained using the eigendecomposition and taking the square root of all the eigenvalues. When the state variables $\mathbf{x}$ satisfy the system,

$$
\mathcal{M} \frac{\mathrm{d} \mathbf{x}}{\mathrm{d} t}=\mathcal{A} \mathbf{x}+\mathbf{f} \quad \text { with } \mathbf{x}(0)=\mathbf{x}_{0}
$$

the state variables $\mathbf{y}=\mathcal{M}^{\frac{1}{2}} \mathbf{x}$ verify

$$
\frac{\mathrm{d} \mathbf{y}}{\mathrm{d} t}=\mathcal{M}^{-\frac{1}{2}} \mathcal{A} \mathcal{M}^{-\frac{1}{2}} \mathbf{y}+\mathcal{M}^{-\frac{1}{2}} \mathbf{f} \quad \text { with } \mathbf{y}(0)=\mathbf{y}_{0}=\mathcal{M}^{\frac{1}{2}} \mathbf{x}_{0} .
$$

The subspace spanned by $\mathcal{V}$ is associated with the subspace spanned by $\mathbf{V}=\mathcal{M}^{\frac{1}{2}} \mathcal{V}$. The reduced-order models have the following identification

$$
\mathbf{x}_{\mathrm{ROM}}=\tilde{\mathbf{x}}+\mathcal{V} \mathbf{x}_{r} \quad \leftrightarrow \quad \mathbf{y}_{\mathrm{ROM}}=\tilde{\mathbf{y}}+\mathbf{V} \mathbf{x}_{r}=\mathcal{M}^{\frac{1}{2}} \mathbf{x}_{\mathrm{ROM}} .
$$

If the error when approximating the state variable $\mathbf{y}$ is decomposed as

$$
\mathbf{e}_{\mathrm{ROM}}=\mathbf{y}-\tilde{\mathbf{y}}-\mathbf{V}\left(\mathbf{V}^{T} \mathbf{V}\right)^{-1} \mathbf{V}^{T}(\mathbf{y}-\tilde{\mathbf{y}})+\mathbf{V}\left[\left(\mathbf{V}^{T} \mathbf{V}\right)^{-1} \mathbf{V}^{T}(\mathbf{y}-\tilde{\mathbf{y}})-\mathbf{x}_{r}\right],
$$


then the error $\mathcal{E}_{\mathrm{ROM}}=\mathbf{x}-\mathbf{x}_{\mathrm{ROM}}$ is written as

$$
\mathcal{E}_{\mathrm{ROM}}=\mathbf{x}-\tilde{\mathbf{x}}-\mathcal{V}\left(\mathcal{V}^{T} \mathcal{M} \mathcal{V}\right)^{-1} \mathcal{V}^{T} \mathcal{M}(\mathbf{x}-\tilde{\mathbf{x}})+\mathcal{V}\left[\left(\mathcal{V}^{T} \mathcal{M V}\right)^{-1} \mathcal{V}^{T} \mathcal{M}(\mathbf{x}-\tilde{\mathbf{x}})-\mathbf{x}_{r}\right]
$$

and, for norms, $\left\|\mathbf{e}_{\mathrm{ROM}}\right\|_{2}=\left\|\mathcal{E}_{\mathrm{ROM}}\right\|_{\mathcal{M}}$ and

$$
\left\|\mathbf{y}-\tilde{\mathbf{y}}-\mathbf{V}\left(\mathbf{V}^{T} \mathbf{V}\right)^{-1} \mathbf{V}^{T}(\mathbf{y}-\tilde{\mathbf{y}})\right\|_{2}=\left\|\mathbf{x}-\tilde{\mathbf{x}}-\mathcal{V}\left(\mathcal{V}^{T} \mathcal{M V}\right)^{-1} \mathcal{V}^{T} \mathcal{M}(\mathbf{x}-\tilde{\mathbf{x}})\right\|_{\mathcal{M}} .
$$

Similarly, the matrix norm $\left\|\mathbf{V}^{T} \mathbf{A} \mathbf{Z}\left(\mathbf{Z}^{T} \mathbf{Z}\right)^{-1} \mathbf{Z}^{T}\right\|_{\left(\mathbf{V}^{T} \mathbf{V}\right)^{-1}}$ satisfies

$$
\left\|\mathbf{V}^{T} \mathbf{A} \mathbf{Z}\left(\mathbf{Z}^{T} \mathbf{Z}\right)^{-1} \mathbf{Z}^{T}\right\|_{\left(\mathbf{V}^{T} \mathbf{V}\right)^{-1}}=\left\|\mathcal{V}^{T} \mathcal{A} \mathcal{Z}\left(\mathcal{Z}^{T} \mathcal{M} \mathcal{Z}\right)^{-1} \mathcal{Z}^{T} \mathcal{M}^{\frac{1}{2}}\right\|_{\left(\mathcal{V}^{T} \mathcal{M V}\right)^{-1}}
$$

where the columns of matrix $\mathcal{Z}$ span the $\mathcal{M}$-orthogonal complement for the span of $\mathcal{V}$. The results for the discrete scheme obtained after using the trapezoidal time integration are derived in a similar way.

To study the proper orthogonal decomposition for this generalized first-order system, a subspace of dimension $k$ is obtained by solving the minimization problem

$$
\operatorname{argmin}_{\operatorname{rank}\{\mathcal{V}\}=k} \int_{0}^{T}\left\|\mathbf{x}(t)-\tilde{\mathbf{x}}(t)-\mathcal{V}\left(\mathcal{V}^{T} \mathcal{M} \mathcal{V}\right)^{-1} \mathcal{V}^{T} \mathcal{M}(\mathbf{x}(t)-\tilde{\mathbf{x}}(t))\right\|_{\mathcal{M}}^{2} \mathrm{~d} t .
$$

The same subspace is obtained by the maximization problem

$$
\operatorname{argmax}_{\operatorname{rank}\{\mathcal{V}\}=k} \int_{0}^{T}\left\|\mathcal{V}\left(\mathcal{V}^{T} \mathcal{M V}\right)^{-1} \mathcal{V}^{T} \mathcal{M}(\mathbf{x}(t)-\tilde{\mathbf{x}}(t))\right\|_{\mathcal{M}}^{2} \mathrm{~d} t
$$

The norm can be written as

$$
\begin{aligned}
& \left\|\mathcal{V}\left(\mathcal{V}^{T} \mathcal{M V}\right)^{-1} \mathcal{V}^{T} \mathcal{M}(\mathbf{x}(t)-\tilde{\mathbf{x}}(t))\right\|_{\mathcal{M}}^{2} \\
& =\operatorname{tr}\left(\mathcal{M V}\left(\mathcal{V}^{T} \mathcal{M V}\right)^{-1} \mathcal{V}^{T} \mathcal{M}(\mathbf{x}(t)-\tilde{\mathbf{x}}(t))(\mathbf{x}(t)-\tilde{\mathbf{x}}(t))^{T} \mathcal{M} \mathcal{V}\left(\mathcal{V}^{T} \mathcal{M V}\right)^{-1} \mathcal{V}^{T}\right) \\
& =\operatorname{tr}\left(\mathcal{V}^{T} \mathcal{M}(\mathbf{x}(t)-\tilde{\mathbf{x}}(t))(\mathbf{x}(t)-\tilde{\mathbf{x}}(t))^{T} \mathcal{M} \mathcal{V}\left(\mathcal{V}^{T} \mathcal{M} \mathcal{V}\right)^{-1}\right)
\end{aligned}
$$

By introducing the matrix

$$
\mathbf{R}=\int_{0}^{T}(\mathbf{x}(t)-\tilde{\mathbf{x}}(t))(\mathbf{x}(t)-\tilde{\mathbf{x}}(t))^{T} \mathrm{~d} t
$$

the norm to maximize becomes

$$
\int_{0}^{T}\left\|\mathcal{V}\left(\mathcal{V}^{T} \mathcal{M V}\right)^{-1} \mathcal{V}^{T} \mathcal{M}(\mathbf{x}(t)-\tilde{\mathbf{x}}(t))\right\|_{\mathcal{M}}^{2}=\operatorname{tr}\left(\mathcal{V}^{T} \mathcal{M} \mathbf{R} \mathcal{M V}\left(\mathcal{V}^{T} \mathcal{M V}\right)^{-1}\right)
$$

A stationary point will satisfy

$$
\mathcal{M} \mathbf{R} \mathcal{M V}\left(\mathcal{V}^{T} \mathcal{M V}\right)^{-1}=\mathcal{M V}\left(\mathcal{V}^{T} \mathcal{M V}\right)^{-1} \mathcal{V}^{T} \mathcal{M} \mathbf{R} \mathcal{M V}\left(\mathcal{V}^{T} \mathcal{M V}\right)^{-1}
$$

indicating that $\mathcal{V}$ is a stable subspace for the pencil $(\mathcal{M R} \mathcal{M}, \mathcal{M})$. Denoting $\left(\lambda_{i}^{\infty}\right)_{1 \leq i \leq r}$ the non-zero eigenvalues of the pencil $(\mathcal{M} \mathbf{R} \mathcal{M}, \mathcal{M})$ - ordered in a non-increasing fashion - the projection error for the minimization problem is

$$
\int_{0}^{T}\left\|\mathbf{x}(t)-\tilde{\mathbf{x}}(t)-\mathcal{V}\left(\mathcal{V}^{T} \mathcal{M V}\right)^{-1} \mathcal{V}^{T} \mathcal{M}(\mathbf{x}(t)-\tilde{\mathbf{x}}(t))\right\|_{\mathcal{M}}^{2} \mathrm{~d} t=\sum_{i=k+1}^{r} \lambda_{i}^{\infty},
$$


where $\mathcal{V}$ is composed of the first $k$ dominant eigenvectors. Similarly, the proper orthogonal decomposition for the discrete snapshots $\mathbf{x}_{j}-\tilde{\mathbf{x}}_{j}$ will study the eigendecomposition for the pencil $(\mathcal{M} \hat{\mathbf{R}} \mathcal{M}, \mathcal{M})$ where the matrix $\hat{\mathbf{R}}$ is

$$
\hat{\mathbf{R}}=\sum_{j=0}^{n_{t}}\left(\mathbf{x}_{j}-\tilde{\mathbf{x}}_{j}\right)\left(\mathbf{x}_{j}-\tilde{\mathbf{x}}_{j}\right)^{T}
$$

\section{A.3. Derivation for Section 3}

If the entire continuous trajectory is available on the interval $[0, T]$, the proper orthogonal decomposition can generate a subspace of dimension $k$ by solving the minimization problem

$$
\operatorname{argmin}_{\operatorname{rank}\{\mathcal{V}\}=k} \int_{0}^{T}\left\|\mathbf{x}(t)-\tilde{\mathbf{x}}(t)-\mathcal{V}\left(\mathcal{V}^{T} \mathcal{M} \mathcal{V}\right)^{-1} \mathcal{V}^{T} \mathcal{M}(\mathbf{x}(t)-\tilde{\mathbf{x}}(t))\right\|_{\mathcal{M}}^{2} \mathrm{~d} t \quad \text { where } \mathbf{x}=\left[\begin{array}{c}
\frac{\mathrm{d} \mathbf{u}}{\mathrm{d} t}(t) \\
\mathbf{u}(t)
\end{array}\right] .
$$

The same subspace is obtained by the maximization problem

$$
\operatorname{argmax}_{\operatorname{rank}\{\mathcal{V}\}=k} \int_{0}^{T}\left\|\mathcal{V}\left(\mathcal{V}^{T} \mathcal{M V}\right)^{-1} \mathcal{V}^{T} \mathcal{M}(\mathbf{x}(t)-\tilde{\mathbf{x}}(t))\right\|_{\mathcal{M}}^{2} \mathrm{~d} t
$$

Rewrite the norm as

$$
\begin{aligned}
& \| \mathcal{V}\left(\mathcal{V}^{T} \mathcal{M} \mathcal{V}\right)^{-1} \mathcal{V}^{T} \mathcal{M}(\mathbf{x}(t)-\tilde{\mathbf{x}}(t)) \|_{\mathcal{M}}^{2} \\
&=\operatorname{tr}\left(\mathcal{M V}\left(\mathcal{V}^{T} \mathcal{M} \mathcal{V}\right)^{-1} \mathcal{V}^{T} \mathcal{M}(\mathbf{x}(t)-\tilde{\mathbf{x}}(t))(\mathbf{x}(t)-\tilde{\mathbf{x}}(t))^{T} \mathcal{M} \mathcal{V}\left(\mathcal{V}^{T} \mathcal{M} \mathcal{V}\right)^{-1} \mathcal{V}^{T}\right) \\
&=\operatorname{tr}\left(\mathcal{V}^{T} \mathcal{M}(\mathbf{x}(t)-\tilde{\mathbf{x}}(t))(\mathbf{x}(t)-\tilde{\mathbf{x}}(t))^{T} \mathcal{M V}\left(\mathcal{V}^{T} \mathcal{M V}\right)^{-1}\right)
\end{aligned}
$$

The inner matrix satisfies

$$
(\mathbf{x}(t)-\tilde{\mathbf{x}}(t))(\mathbf{x}(t)-\tilde{\mathbf{x}}(t))^{T}=\left[\begin{array}{cc}
\left(\frac{\mathrm{du}}{\mathrm{d} t}(t)-\frac{\mathrm{d} \tilde{\mathbf{u}}}{\mathrm{d} t}(t)\right)\left(\frac{\mathrm{d} \mathbf{u}}{\mathrm{d} t}(t)-\frac{\mathrm{d} \tilde{\mathbf{u}}}{\mathrm{d} t}(t)\right)^{T} & \left(\frac{\mathrm{d} \mathbf{u}}{\mathrm{d} t}(t)-\frac{\mathrm{d} \tilde{\mathbf{u}}}{\mathrm{d} t}(t)\right)(\mathbf{u}(t)-\tilde{\mathbf{u}}(t))^{T} \\
(\mathbf{u}(t)-\tilde{\mathbf{u}}(t))\left(\frac{\mathrm{du}}{\mathrm{d} t}(t)-\frac{\mathrm{d} \tilde{\mathbf{u}}}{\mathrm{d} t}(t)\right)^{T} & (\mathbf{u}(t)-\tilde{\mathbf{u}}(t))(\mathbf{u}(t)-\tilde{\mathbf{u}}(t))^{T}
\end{array}\right] .
$$

Since the matrices $\mathcal{V}$ and $\mathcal{M}$ are diagonal, the norm becomes

$$
\int_{0}^{T}\left\|\mathcal{V}\left(\mathcal{V}^{T} \mathcal{M} \mathcal{V}\right)^{-1} \mathcal{V}^{T} \mathcal{M}(\mathbf{x}(t)-\tilde{\mathbf{x}}(t))\right\|_{\mathcal{M}}^{2} \mathrm{~d} t=\operatorname{tr}\left(\hat{\mathbf{V}}^{T} \mathbf{M R M} \hat{\mathbf{V}}\left(\hat{\mathbf{V}}^{T} \mathbf{M} \hat{\mathbf{V}}\right)^{-1}\right)
$$

where the matrix $\mathbf{R}$ is defined as

$$
\mathbf{R}=\int_{0}^{T}\left(\frac{\mathrm{d} \mathbf{u}}{\mathrm{d} t}(t)-\frac{\mathrm{d} \tilde{\mathbf{u}}}{\mathrm{d} t}(t)\right)\left(\frac{\mathrm{d} \mathbf{u}}{\mathrm{d} t}(t)-\frac{\mathrm{d} \tilde{\mathbf{u}}}{\mathrm{d} t}(t)\right)^{T}+\frac{1}{\tau^{2}}(\mathbf{u}(t)-\tilde{\mathbf{u}}(t))(\mathbf{u}(t)-\tilde{\mathbf{u}}(t))^{T} \mathrm{~d} t .
$$

A stationary point will satisfy

$$
\operatorname{MRM\hat {\mathbf {V}}}\left(\hat{\mathbf{V}}^{T} \mathbf{M} \hat{\mathbf{V}}\right)^{-1}=\mathbf{M} \hat{\mathbf{V}}\left(\hat{\mathbf{V}}^{T} \mathbf{M} \hat{\mathbf{V}}\right)^{-1} \hat{\mathbf{V}}^{T} \operatorname{MRM\hat {\mathbf {V}}}\left(\hat{\mathbf{V}}^{T} \mathbf{M} \hat{\mathbf{V}}\right)^{-1}
$$

indicating that $\hat{\mathbf{V}}$ is a stable subspace for the pencil (MRM, M). Denoting $\left(\lambda_{i}^{\infty}\right)_{1 \leq i \leq r}$ the non-zero eigenvalues of the pencil (MRM, M) - ordered in a non-increasing fashion - the projection error for the minimization problem is

$$
\begin{aligned}
\int_{0}^{T} \frac{1}{\tau^{2}} \| \mathbf{u}(t)-\tilde{\mathbf{u}}(t)-\hat{\mathbf{V}} & \left(\hat{\mathbf{V}}^{T} \mathbf{M} \hat{\mathbf{V}}\right)^{-1} \hat{\mathbf{V}}^{T} \mathbf{M}(\mathbf{u}(t)-\tilde{\mathbf{u}}(t)) \|_{\mathbf{M}}^{2} \mathrm{~d} t \\
& +\int_{0}^{T}\left\|\left(\frac{\mathrm{d} \mathbf{u}}{\mathrm{d} t}(t)-\frac{\mathrm{d} \tilde{\mathbf{u}}}{\mathrm{d} t}(t)\right)-\hat{\mathbf{V}}\left(\hat{\mathbf{V}}^{T} \mathbf{M} \hat{\mathbf{V}}\right)^{-1} \hat{\mathbf{V}}^{T} \mathbf{M}\left(\frac{\mathrm{d} \mathbf{u}}{\mathrm{d} t}(t)-\frac{\mathrm{d} \tilde{\mathbf{u}}}{\mathrm{d} t}(t)\right)\right\|_{\mathbf{M}}^{2} \mathrm{~d} t=\sum_{i=k+1}^{r} \lambda_{i}^{\infty}
\end{aligned}
$$


Acknowledgements. D. Amsallem acknowledges the support from Office of Naval Research under Grant N00014-11-10707. U. Hetmaniuk acknowledges the support from Office of Naval Research under Grant N00014-11-1-0710. The authors thank the anonymous referees for comments that led to improvements of the manuscript.

\section{REFERENCES}

[1] D. Amsallem and C. Farhat, An interpolation method for adapting reduced-order models and application to aeroelasticity. AIAA J. 46 (2008) 1803-1813.

[2] D. Amsallem, J. Cortial, K. Carlberg and C. Farhat, A method for interpolating on manifolds structural dynamics reduced-order models. Int. J. Numer. Methods Eng. 80 (2009) 1241-1258.

[3] D. Amsallem, J. Cortial and C. Farhat, Toward real-time computational-fluid-dynamics-based aeroelastic computations using a database of reduced-order information. AIAA J. 48 (2010) 2029-2037.

[4] D. Amsallem and J. Roychowdhury, ModSpec: An open, flexible specification framework for multi-domain device modelling. 2011 IEEE/ACM International Conference on Computer-Aided Design (ICCAD) (2011) 367-374.

[5] D. Amsallem and C. Farhat, On the stability of linearized reduced-order models: descriptor vs non-descriptor form. 42nd AIAA Fluid Dynamics Conference and Exhibit (2012) 25-28 New Orleans, LA (2012).

[6] A. Antoulas, Approximation of large-scale dynamical systems. SIAM, Philadelphia (2005).

[7] C. Beattie and S. Gugercin, Krylov-based model reduction of second-order systems with proportional damping, in Proc. 44th $C D C / E C C$ (2005) 2278-2283.

[8] C. Beattie and S. Gugercin, Interpolatory projection methods for structure-preserving model reduction. Systems Control Lett. 58 (2009) 225-232.

[9] T. Bui-Thanh, M. Damodoran and K. Willcox, Aerodynamic data reconstruction and inverse design using proper orthogonal decomposition. AIAA J. 42 (2004) 1505-1516.

[10] D. Chapelle, A. Gariah and J. Sainte-Marie, Galerkin approximation with proper orthogonal decomposition: new error estimates and illustrative examples. ESAIM: M2AN 46 (2012) 731-757.

[11] S. Chaturantabut and D. Sorensen, A state space error estimate for POD-DEIM nonlinear model reduction. SIAM J. Numer. Anal. 50 (2012) 46-63.

[12] R. Guyan, Reduction of stiffness and mass matrices. AIAA J. 3 (1965) 380-380.

[13] S. Han and B. Feeny. Enhanced proper orthogonal decomposition for the modal analysis of homogeneous structures. J. Vibration Control 8 (2002) 19-40.

[14] S. Herkt, M. Hinze and R. Pinnau, Convergence analysis of Galerkin POD for linear second order evolution equations. Hamburger Beiträge zur Angewandten Math. 2011-06 (2011).

[15] U. Hetmaniuk and R. Lehoucq, Uniform accuracy of eigenpairs from a shift-invert Lanczos method. SIAM J. Matrix Anal. Appl. 28 (2006) 927-948.

[16] C. Homescu, L. Petzold and R. Serban, Error estimation for reduced-order models of dynamical systems. SIAM Rev. 49 (2007) $277-299$.

[17] T. Hughes, The finite element method: linear static and dynamic finite element analysis. Prentice-Hall (1987).

[18] D. B. Huynh, D. Knezevic and A. Patera, A Laplace transform certified reduced basis method; application to the heat equation and wave equation. C.R. Acad. Sci. Paris, Série I 349 (2011) 401-405.

[19] K. Karhunen, Zur Spektraltheorie Stochastischer Prozesse. Ann. Acad. Sci. Fennicae 34 (1946).

[20] G. Kerschen, J.C. Golinval, A. Vakakis and L. Bergman, The method of proper orthogonal decomposition for dynamical characterization and order reduction of mechanical systems: an overview. Nonlinear Dyn. 41 (2005) 147-169.

[21] K. Kunisch and S. Volkwein, Galerkin proper orthogonal decomposition methods for parabolic problems. Numer. Math. 90 (2001) 117-148.

[22] K. Kunisch and S. Volkwein, Galerkin proper orthogonal decomposition methods for a general equation in fluid dynamics. SIAM J. Numer. Anal. 40 (2002) 492-515.

[23] K. Kunisch and S. Volkwein, Crank-Nicholson Galerkin proper orthogonal decomposition approximations for a general equation in fluid dynamics. 18th GAMM Seminar on Multigrid and Related Methods for Optimization Problems, Leipzig (2002) 97-114.

[24] K. Kunisch and S. Volkwein, Optimal snapshot location for computing POD basis functions. ESAIM: M2AN 44 (2010) 509-529.

[25] O. Lass and S. Volkwein. Adaptive POD basis computation for parameterized nonlinear systems using optimal snapshot location. Konstanzer Schriften Math. 304 (2012) 1-27.

[26] J. Lienemann, D. Billger, E. B. Rudnyi, A. Greiner and J.G. Korvink, MEMS compact modeling meets model order reduction: examples of the application of Arnoldi methods to microsystems devices. Technical Proceedings of the 2004 Nanotechnology conference and trade show, Nanotech 2004, March 1-7, Boston, MA 2 (2004) 303-306.

[27] T. Lieu and C. Farhat, Adaptation of aeroelastic reduced-order models and application to an F-16 configuration. AIAA J. 45 (2007) 1244-1269.

[28] M. Loeve. Fonctions aléatoires de second ordre. C.R. Acad. Sci. Paris, 220 (1945).

[29] Oberwolfach benchmark collection. (2005). Available at http://portal.uni-freiburg.de/imteksimulation/downloads/ benchmark/.

[30] A. Quarteroni, R. Sacco, and F. Saleri, Numerical Mathematics, Number 37 in Texts in Applied Mathematics. Springer (2000). 
[31] M. Rathinam and L. Petzold, A new look at proper orthogonal decomposition. SIAM J. Numer. Anal. 41 (2003) $1893-1925$.

[32] E. W. Sachs and M. Schu, A priori error estimates for reduced order models in finance. ESAIM: M2AN. Doi: $10.1051 / \mathrm{m} 2 \mathrm{na} / 2012039$.

[33] L. Sirovich, Turbulence and the dynamics of coherent structures. Parts I-II. Quarterly of Applied Mathematics XVL (1987) $561-590$.

[34] A. Tan, Reduced basis methods for 2nd order wave equation: application to one dimensional seismic problem. Masters thesis, Singapore-MIT Alliance, National University of Singapore (2006).

[35] J.P. Thomas, E. Dowell and K. Hall, Three-dimensional transonic aeroelasticity using proper orthogonal decomposition-based reduced order models. J. Aircraft 40 (2003) 544-551.

[36] K. Veroy, C. Prud'homme, D. Rovas, and A. Patera, A posteriori error bounds for reduced-basis approximation of parametrized noncoercive and nonlinear elliptic partial differential equations. AIAA Pap. 2003-3847 (2003).

[37] K. Veroy and A. Patera, Certified real-time solution of the parametrized steady incompressible Navier-Stokes equations: rigorous reduced-based a posteriori error bounds. Int. J. Numer. Methods Eng. 47 (2005) 773-788.

[38] S. Volkwein, Model reduction using proper orthogonal decomposition. Lect. Notes (2011) 1-43. Available at http://www.math. uni-konstanz.de/numerik/personen/volkwein/teaching/POD-Vorlesung.pdf. 